\title{
CONVERGENCE OF THE PENALTY METHOD APPLIED TO A CONSTRAINED CURVE STRAIGHTENING FLOW*
}

\author{
DIETMAR OELZ
}

\begin{abstract}
We apply the penalty method to the curve straightening flow of inextensible planar open curves generated by the Kirchhoff bending energy. Thus we consider the curve straightening flow of extensible planar open curves generated by a combination of the Kirchhoff bending energy and a functional penalizing deviations from unit arc-length.

We start with the governing equations of the explicit parametrization of the curve and derive an equivalent system for the two quantities indicatrix and arc-length. We prove existence and regularity of solutions and use the indicatrix/arc-length representation to compute the energy dissipation. We prove its coercivity and conclude exponential decay of the energy.

Finally, by an application of the Lions-Aubin Lemma, we prove convergence of solutions to a limit curve which is the solution of an analogous gradient flow on the manifold of inextensible open curves. This procedure also allows us to characterize the Lagrange multiplier in the limit model as a weak limit of force terms present in the relaxed model.
\end{abstract}

Key words. Curve straightening flow, energy dissipation, elastic regularization, curvature flow, penalty method.

AMS subject classifications. 53C44, 35K91, 92C10.

\section{Introduction}

On the set of planar, open curves

$$
\mathcal{A}:=\left\{z \in H^{2}\left((0,1), \mathbb{R}^{2}\right) ; b_{\min }<\left|z^{\prime}\right|<b_{\max }\right\},
$$

where $b_{\min }, b_{\max } \in \mathbb{R}$ with $0<b_{\min }<1<b_{\max }$, we consider the family of gradient flows generated by the potential

$$
\mathcal{E}_{\varepsilon}[z]:=\mathcal{E}^{\text {curv }}[z]+\mathcal{E}_{\varepsilon}^{\text {pen }}[z]
$$

The potential consists of a functional for the curvature energy

$$
\mathcal{E}^{\mathrm{curv}}[z]:=\int_{0}^{1} \frac{\left|z^{\prime \prime}\right|^{2}}{2} d s,
$$

and of a penalizing potential

$$
\mathcal{E}_{\varepsilon}^{\text {pen }}[z]:=\frac{1}{\varepsilon} \int_{0}^{1} E\left(\left|z^{\prime}\right|\right) d s,
$$

where

$$
E \in \mathcal{C}^{2}\left(\left(b_{\min }, b_{\max }\right), \mathbb{R}_{+}\right) \quad \text { with }\left\{\begin{array}{l}
\lim _{x \rightarrow b_{\min }} E(x)=\lim _{x \rightarrow b_{\max }} E(x)=\infty, \\
E^{\prime \prime}(x) \geq \kappa>0 \quad \forall \quad x \in\left(b_{\min }, b_{\max }\right), \\
E^{\prime}(1)=E(0)=0 .
\end{array}\right.
$$

*Received: November 17, 2012; accepted (in revised form): April 24, 2013. Communicated by Richard Tsai.

${ }^{\dagger}$ Johann Radon Institute for Computational and Applied Mathematics, Apostelgasse 23, A 1030 Wien, Austria (dietmar.oelz@oeaw.ac.at). 
Hence $E=E(x)$ is a strictly convex $\mathcal{C}^{2}$-function which takes its minimum at $x=1$. One possible choice is $E(x)=\frac{(x-1)^{2}}{(x-1 / 2)(4-x)}$. We use the functional (1.3) to penalize deviations from unit arc-length with a view to enforce unit arc-length by letting the scaling parameter $\varepsilon$ tend to zero.

The gradient flow generated by the functional (1.1) is described by the system

$$
\left\{\begin{array}{l}
\partial_{t} z_{\varepsilon}+z_{\varepsilon}^{\prime \prime \prime \prime}-\left(\frac{1}{\varepsilon} E^{\prime}\left(\left|z_{\varepsilon}^{\prime}\right|\right) \frac{z_{\varepsilon}^{\prime}}{\left|z_{\varepsilon}^{\prime}\right|}\right)^{\prime}=0, \\
z_{\varepsilon}^{\prime \prime}, z_{\varepsilon}^{\prime \prime \prime}-\left.\frac{1}{\varepsilon} E^{\prime}\left(\left|z_{\varepsilon}^{\prime}\right|\right) \frac{z_{\varepsilon}^{\prime}}{\left|z_{\varepsilon}^{\prime}\right|}\right|_{s=0,1}=0, \\
z_{\varepsilon}(t=0, .)=z_{I}(.),
\end{array}\right.
$$

where $z_{I} \in \mathcal{A}$ represents the initial datum of the evolution. Here and throughout the paper ' denotes derivatives with respect to the arc-length. With respect to this system the center of mass $\int_{0}^{1} z_{\varepsilon} d s$ is a conserved quantity.

We show in this paper that the solution $z_{\varepsilon}$ converges to a solution of the gradient flow generated by the Kirchhoff bending energy $(1.2)$ only (cp. $[7,5])$ on the set of open, inextensible curves $\mathcal{A}_{0}:=\left\{u \in H^{2}\left((0,1), \mathbb{R}^{2}\right):\left|u^{\prime}\right| \equiv 1\right\}$ described by the system

$$
\left\{\begin{array}{l}
\partial_{t} z_{0}+z_{0}^{\prime \prime \prime \prime}-\left(\lambda_{0} z_{0}^{\prime}\right)^{\prime}=0, \\
\left.z_{0}^{\prime \prime}\right|_{s=0,1}=0, \\
z_{0}^{\prime \prime \prime}-\left.\lambda_{0} z^{\prime}\right|_{s=0,1}=0, \\
\left|z_{0}^{\prime}\right|=1 \\
z_{0}(t=0, .)=z_{I}(.) .
\end{array}\right.
$$

Here $\lambda_{0}=\lambda_{0}(t, s) \in \mathbb{R}$ is a Lagrange multiplier function determined by the constraint on the arc-length

$$
\left|z_{0}^{\prime}\right| \equiv 1
$$

The system (1.5) can be seen as a regularization of the limit system (1.6) which is treated in detail in [9]. The results of this previous study are summarized in Section 2. The limit model exhibits analytical properties like long time convergence at an exponential rate which, as it turns out, can be to a large extent generalized to the approximating model. As a matter of fact a large part of this study is devoted to generalizing the findings on energy dissipation and large time convergence. This provides the necessary a priori bounds to prove compactness to pass to the limit as $\varepsilon \rightarrow 0$.

The system (1.5) can be used as a numerical approximation to the limit model which has appeared in the modeling of actin-filaments in biological cells (cf. $[10,11])$. Most notably in combination with an augmented Lagrangian approach the penalizing potential is currently being used in the development of numerical schemes for models in cellular biophysics.

Furthermore the results of this study provide a characterization of the Lagrange multiplier $\lambda_{0}$ in the limit model (1.6) as a weak limit of a sum of forces, namely the variation of the total energy (1.1) in the direction of the arclength (compare the second line in (1.12), (4.4), and Theorem 1.5). Hence the variations of both the curvature functional (1.2) and the penalizing potential (1.3) contribute to the expression for which we show convergence to the Lagrange multiplier. 
Finally it is also worth mentioning that the present study implies existence of solutions and long time convergence also for the limit problem, although these results were obtained directly in a separate study $([9])$.

For a short review on existing literature on curve straightening flows we refer to [9]. Penalization is a popular method in optimization (e.g. [6]) and control theory (e.g. [3]). One of the few papers we found which studies the application of the penalty method to a constrained evolution problem is [2], although this is done in a totally different context.

The whole argument is based on an approach by which we rewrite the system (1.5) using is based on an approach by which we rewrite the system (1.5) using the following notation. The symbol $b_{\varepsilon}=b_{\varepsilon}(t, s)$ denotes the arc-length

$$
b_{\varepsilon}:=\left|z_{\varepsilon}^{\prime}\right|,
$$

and $\omega_{\varepsilon}=\omega_{\varepsilon}(t, s) \in \mathbb{R}$ represents the "indicatrix" of the curve $z_{\varepsilon}$ (e.g. see [8]) so that

$$
\frac{z_{\varepsilon}^{\prime}}{\left|z_{\varepsilon}^{\prime}\right|}=\left(\cos \left(\omega_{\varepsilon}\right), \sin \left(\omega_{\varepsilon}\right)\right)
$$

The reconstruction of the curve $z_{\varepsilon}$ from the arc-length $b_{\varepsilon}$ and the indicatrix $\omega_{\varepsilon}$ has to be done in such a way, that the center of mass of the initial datum $\bar{z}_{I}:=\int_{0}^{1} z_{I} d s \in \mathbb{R}^{2}$ is conserved,

$$
z_{\varepsilon}(t, s)=\bar{z}_{I}-\int_{0}^{1} \int_{0}^{\tilde{s}}\left(\begin{array}{c}
\cos \left(\omega_{\varepsilon}(t, \bar{s})\right) \\
\sin \left(\omega_{\varepsilon}(t, \bar{s})\right)
\end{array}\right) b_{\varepsilon} d \bar{s} d \tilde{s}+\int_{0}^{s}\left(\begin{array}{c}
\cos \left(\omega_{\varepsilon}(t, \bar{s})\right) \\
\sin \left(\omega_{\varepsilon}(t, \bar{s})\right)
\end{array}\right) d \bar{s} .
$$

Observe also that $b_{\varepsilon} \omega_{\varepsilon}^{\prime}$ and $b_{\varepsilon}^{\prime}$ are both orthogonal components of $z_{\varepsilon}^{\prime \prime}$ since

$$
\omega_{\varepsilon}^{\prime}=\frac{z_{\varepsilon}^{\prime \perp}}{\left|z_{\varepsilon}^{\prime}\right|^{2}} \cdot z_{\varepsilon}^{\prime \prime} \quad \text { and } \quad b_{\varepsilon}^{\prime}=\frac{z_{\varepsilon}^{\prime}}{\left|z_{\varepsilon}^{\prime}\right|} \cdot z_{\varepsilon}^{\prime \prime}
$$

and that the total energy (1.1) can be written as

$$
\mathcal{E}_{\varepsilon}[z]=\int_{0}^{1}\left(\frac{1}{2}\left(b \omega^{\prime}\right)^{2}+\frac{1}{2}\left(b^{\prime}\right)^{2}+\frac{1}{\varepsilon} E(b)\right) d s,
$$

where $\omega$ and $b$ are the indicatrix and an arc-length of the curve $z$, respectively. Also the initial datum $z_{I}$ will be occasionally written in terms of its indicatrix $\omega_{I}$ and its arc-length $b_{I}$, which allows us to give two alternative expressions for the initial energy:

$$
\mathcal{E}_{I}:=\int_{0}^{1}\left[\frac{1}{2}\left|z_{I}^{\prime \prime}\right|^{2}+\frac{1}{\varepsilon} E\left(\left|z_{I}^{\prime}\right|\right)\right] d s=\int_{0}^{1}\left[\frac{1}{2}\left(b_{I} \omega_{I}^{\prime}\right)^{2}+\frac{1}{2}\left(b_{I}^{\prime}\right)^{2}+\frac{1}{\varepsilon} E\left(b_{I}\right)\right] d s .
$$

Furthermore, in view of (1.5), we use the following notation for what below will be shown to be the scalar product of $z_{\varepsilon}^{\prime \prime \prime}-\frac{1}{\varepsilon} E^{\prime}\left(\left|z_{\varepsilon}^{\prime}\right|\right) \frac{z^{\prime}}{\left|z^{\prime}\right|}$ with $\frac{z^{\prime \prime}}{\left|z^{\prime}\right|}$ and $\frac{z^{\prime}}{\left|z^{\prime}\right|}$ respectively, namely

$$
\begin{aligned}
r_{\varepsilon} & :=\frac{1}{b_{\varepsilon}}\left(b_{\varepsilon}^{2} \omega_{\varepsilon}^{\prime}\right)^{\prime}, \\
\lambda_{\varepsilon} & :=b_{\varepsilon}\left(\omega_{\varepsilon}^{\prime}\right)^{2}-b_{\varepsilon}^{\prime \prime}+\frac{1}{\varepsilon} E^{\prime}\left(b_{\varepsilon}\right) .
\end{aligned}
$$


The system (1.5) is then equivalent to

$$
\left\{\begin{array}{l}
\partial_{t} \omega_{\varepsilon}+\left(r_{\varepsilon}^{\prime \prime}-r_{\varepsilon}\left(\omega_{\varepsilon}^{\prime}\right)^{2}-\lambda_{\varepsilon}^{\prime} \omega_{\varepsilon}^{\prime}-\left(\lambda_{\varepsilon} \omega_{\varepsilon}^{\prime}\right)^{\prime}\right) \frac{1}{b_{\varepsilon}}=0 \\
\partial_{t} b_{\varepsilon}-r_{\varepsilon}^{\prime} \omega_{\varepsilon}^{\prime}-\left(r_{\varepsilon} \omega_{\varepsilon}^{\prime}\right)^{\prime}-\lambda_{\varepsilon}^{\prime \prime}+\left(\omega_{\varepsilon}^{\prime}\right)^{2} \lambda_{\varepsilon}=0 \\
\omega_{\varepsilon}^{\prime}, \omega_{\varepsilon}^{\prime \prime},\left.\lambda_{\varepsilon}\right|_{s=0,1}=0 \\
\omega_{\varepsilon}(t=0, .)=\omega_{I}(.) .
\end{array}\right.
$$

With respect to the energy dissipation equality

$$
\frac{d}{d t} \mathcal{E}_{\varepsilon}=-\mathcal{D}_{\varepsilon}
$$

this formulation allows us to derive in a straightforward way that the energy dissipation is given by the two equivalent expressions

$$
\mathcal{D}_{\varepsilon}:=\int_{0}^{1}\left[\left(\omega_{\varepsilon}^{\prime} r_{\varepsilon}+\lambda_{\varepsilon}^{\prime}\right)^{2}+\left(r_{\varepsilon}^{\prime}-\omega_{\varepsilon}^{\prime} \lambda_{\varepsilon}\right)^{2}\right] d s=\int_{0}^{1}\left(z_{\varepsilon}^{\prime \prime \prime \prime}-\left(\frac{1}{\varepsilon} E^{\prime}\left(\left|z_{\varepsilon}^{\prime}\right|\right) \frac{z_{\varepsilon}^{\prime}}{\left|z_{\varepsilon}^{\prime}\right|}\right)^{\prime}\right)^{2} d s,
$$

which will be the main tool for the convergence proof. For later use we introduce a short notation for the two components of the energy dissipation,

$$
m_{\varepsilon}:=\omega_{\varepsilon}^{\prime} r_{\varepsilon}+\lambda_{\varepsilon}^{\prime} \quad \text { and } \quad n_{\varepsilon}:=r_{\varepsilon}^{\prime}-\omega_{\varepsilon}^{\prime} \lambda_{\varepsilon}
$$

which allows us to reformulate the system (1.13) as

$$
\left\{\begin{array}{l}
\partial_{t} \omega_{\varepsilon}=\frac{1}{b_{\varepsilon}}\left(\omega_{\varepsilon}^{\prime} m_{\varepsilon}-n_{\varepsilon}^{\prime}\right) \\
\partial_{t} b_{\varepsilon}=\omega_{\varepsilon}^{\prime} n_{\varepsilon}+m_{\varepsilon}^{\prime} \\
\omega_{\varepsilon}^{\prime}, \omega_{\varepsilon}^{\prime \prime},\left.\lambda_{\varepsilon}\right|_{s=0,1}=0 \\
\omega_{\varepsilon}(t=0, .)=\omega_{I}(.)
\end{array}\right.
$$

In [9] it was shown that the system (1.6) is equivalent to the system

$$
\left\{\begin{array}{l}
\partial_{t} \omega_{0}+\omega_{0}^{\prime \prime \prime \prime}-\omega_{0}^{\prime 2} \omega^{\prime \prime}-\left(\omega_{0}^{\prime \prime} \lambda_{0}+2 \omega_{0}^{\prime} \lambda_{0}^{\prime}\right)=0, \\
-\lambda_{0}^{\prime \prime}+\omega_{0}^{\prime 2} \lambda_{0}=\omega_{0}^{\prime \prime \prime} \omega_{0}^{\prime}+\left(\omega_{0}^{\prime \prime} \omega_{0}^{\prime}\right)^{\prime}, \\
\omega_{0}^{\prime}, \omega_{0}^{\prime \prime},\left.\lambda_{0}\right|_{s=0,1}=0 \\
\omega_{0}(t=0, .)=\omega_{I}(.)
\end{array}\right.
$$

where $\omega_{0}$ represents the "indicatrix" of the curve $z_{0}$. It was also shown in this study that the curvature energy of solutions to system (1.6) decays at an exponential rate larger or equal to $2 \pi^{4}$ and that limit curves are straight lines.

The paper will be structured as follows. In Section 2 we summarize the results of [9] as they are used in the present paper.

In Section 3 we derive the system (1.5) and prove that as a result of the usual construction in the theory of gradient flows and steepest descent flows (cp. [4, 1]) there exists a weak solution $z_{\varepsilon}$ to the system (1.5).

THEOREM 1.1. If $z_{I} \in \mathcal{A}$, then there is $z_{\varepsilon} \in H_{t, \text { loc }}^{1} L_{s}^{2} \cap \mathcal{C}_{t}^{0, \frac{1}{2}} L_{s}^{2} \cap \mathcal{C}_{t}^{0, \frac{1}{8}} \mathcal{C}_{s}^{1} \cap L_{t}^{\infty} H_{s}^{2}$ such that $z_{\varepsilon}$ is a weak solution of (1.5) satisfying

$$
\int_{0}^{\infty} \int_{0}^{1}\left[\partial_{t} z_{\varepsilon} \cdot v+z_{\varepsilon}^{\prime \prime} \cdot v^{\prime \prime}+\frac{1}{\varepsilon} E^{\prime}\left(\left|z_{\varepsilon}^{\prime}\right|\right) \frac{z_{\varepsilon}^{\prime}}{\left|z_{\varepsilon}^{\prime}\right|} \cdot v^{\prime}\right] d s d t=0
$$


for all $v \in \mathcal{C}_{c}^{\infty}\left(\mathbb{R}_{+}, \mathcal{C}_{s}^{\infty}\right)$ and $z_{\varepsilon}(t=0,)=.z_{I}$. It holds that $\partial_{t} z_{\varepsilon} \in L_{t}^{2} L_{s}^{2}$ with $\left\|\partial_{t} z_{\varepsilon}\right\|_{L_{t}^{2} L_{s}^{2}} \leq$ $\sqrt{2 \mathcal{E}_{I}}$.

Here and in the sequel we abbreviate the notation of function spaces writing the subscripts

$$
\begin{array}{lll}
\square_{t} \quad \text { for function spaces on } & \{t \in[0, \infty)\}, & \text { and } \\
\square_{s} \quad \text { for function spaces on } & \{s \in[0,1]\} .
\end{array}
$$

In Section 4 we rewrite (1.5) obtaining the system (1.13) and prove the following theorem.

THEOREM 1.2. The solution according to Theorem 1.1 gives a distributional sense to the system (1.13), i.e. for $\omega_{\varepsilon}$ being the indicatrix of $z_{\varepsilon}$ and $b_{\varepsilon}$ its arc-length, and using the notation (1.12), it holds that

$$
\begin{aligned}
& \int_{0}^{\infty} \int_{0}^{1}\left[-b_{\varepsilon} \phi_{t}+\left(r_{\varepsilon} \omega_{\varepsilon}^{\prime}+\lambda_{\varepsilon}^{\prime}\right) \phi^{\prime}+\left(\omega_{\varepsilon}^{\prime} \lambda_{\varepsilon}-r_{\varepsilon}^{\prime}\right) \omega_{\varepsilon}^{\prime} \phi\right] d s d t=0 \\
& \int_{0}^{\infty} \int_{0}^{1}\left[-\omega_{\varepsilon} \partial_{t} \psi-\left(r_{\varepsilon}^{\prime}-\lambda_{\varepsilon} \omega_{\varepsilon}^{\prime}\right)\left(\frac{\psi}{b_{\varepsilon}}\right)^{\prime}-\left(r_{\varepsilon} \omega_{\varepsilon}^{\prime}+\lambda_{\varepsilon}^{\prime}\right) \frac{\omega_{\varepsilon}^{\prime}}{b_{\varepsilon}} \psi\right] d s d t=0
\end{aligned}
$$

for all $\psi, \phi \in H_{0, t}^{1} L_{s}^{2} \cap L_{t}^{2} H_{0, s}^{1}$ and $\lambda_{\varepsilon}, \omega_{\varepsilon}^{\prime},\left.\omega_{\varepsilon}^{\prime \prime}\right|_{0,1}=0$ a.e. on $\mathbb{R}_{+}$. Furthermore it holds that $\omega_{\varepsilon}, b_{\varepsilon} \in \mathcal{C}_{t}^{0,1 / 8} \mathcal{C}_{s}^{0} \cap L_{t}^{\infty} H_{s}^{1}$ and $\lambda_{\varepsilon}, r_{\varepsilon} \in L_{t}^{2} H_{s}^{1}$. For every $T>0$ it also holds that $\omega_{\varepsilon}^{\prime \prime \prime}, b_{\varepsilon}^{\prime \prime} \in L^{2}\left(0, T ; L_{s}^{1}\right)$ uniformly with respect to $\varepsilon$.

Next, in Section 5, we show the formal derivation of the energy dissipation (1.15) and show that the energy dissipation equation (1.14) is satisfied in a weak sense.

TheOREM 1.3. Let $z_{I} \in \mathcal{A}$, let $z_{\varepsilon}$ be a solution of problem (1.5) according to Theorem 1.1 and let $\left(\omega_{\varepsilon}, b_{\varepsilon}, \lambda_{\varepsilon}\right)$ be the corresponding solution to (1.13) according to Theorem 1.2. Then the energy dissipation equality (1.14) holds weakly in time.

Finally in Section 6 we prove coercivity of (1.15) with respect to the total energy given by (1.1) and (1.10) (a Poincaré type inequality), obtaining the exponential decay of the energy.

TheOREM 1.4. (Poincaré type inequality) Under the assumptions of Theorem 1.3 there is a constant $C>0$ such that

$$
\mathcal{E}_{\varepsilon} \leq \mathcal{E}_{I} \exp (-C t)
$$

Most notably at large times the total energy tends to zero, $\lim _{t \rightarrow \infty} \mathcal{E}_{\varepsilon}=0$.

Finally we obtain the main theorem of this paper in Section 7. It states the convergence as $\varepsilon \rightarrow 0$ and the consistency with the limit system, i.e. a subsequence of solutions to the problem (1.13) and (1.5) converges to a solution of (1.18) and (1.6), respectively.

Theorem 1.5. Let $\left(\omega_{\varepsilon}, b_{\varepsilon}, \lambda_{\varepsilon}\right)$ as in Theorem 1.2 be a solution to the system (1.13). Then there is a subsequence $\varepsilon_{i} \rightarrow 0$ and limit functions $\left(\omega_{0}, \lambda_{0}\right)$ such that

$$
\begin{array}{ccc}
b_{\varepsilon_{i}} \rightarrow 1 \quad \text { in } \quad L^{1}\left((0, T), W_{s}^{1, r}\right) \cap L^{q}\left((0, T), H_{s}^{1}\right) & (1 \leq r, q<\infty), \\
\omega_{\varepsilon_{i}} \rightarrow \omega_{0} \quad \text { in } \quad L^{1}\left((0, T), W_{s}^{2, r}\right) \cap L^{q}\left((0, T), H_{s}^{1}\right) \quad(1 \leq r, q<\infty),
\end{array}
$$




$$
\begin{aligned}
m_{\varepsilon_{i}} & \rightarrow \omega_{0}^{\prime} \omega_{0}^{\prime \prime}+\lambda_{0}^{\prime} \quad \text { in } L_{t}^{2} L_{s}^{2}, \\
n_{\varepsilon_{i}} & \rightarrow \omega_{0}^{\prime \prime \prime}-\omega_{0}^{\prime} \lambda_{0} \text { in } L_{t}^{2} L_{s}^{2}, \\
r_{\varepsilon_{i}} & \rightarrow \omega_{0}^{\prime \prime} \text { in } L^{1}\left((0, T), L_{s}^{2}\right) \quad \text { and } r_{\varepsilon_{i}}^{\prime} \rightarrow \omega_{0}^{\prime \prime \prime} \text { in } L_{t}^{2} L_{s}^{2}, \\
\lambda_{\varepsilon_{i}} & \rightarrow \lambda_{0} \text { in } L_{t}^{2} H_{s}^{1},
\end{aligned}
$$

as $\varepsilon_{i} \rightarrow 0$, where we use the following notation for constant functions: $1:[0, \infty] \times$ $[0,1] \mapsto \mathbb{R}, x \mapsto 1$ and $\mathbf{0}:[0, \infty] \times[0,1] \mapsto \mathbb{R}, x \mapsto 0$.

The limit functions $\omega_{0}$ and $\lambda_{0}$ satisfy the weak formulation of (1.18) and they also allow us to reconstruct $z_{0}$, which is then a weak solution of (1.6).

\section{Preliminary and technical results}

We cite here some of the results we obtained in [9] for the limit system (1.6) and the equivalent system satisfied by the indicatrix (1.18). We define the constrained set of curves

$$
\mathcal{A}_{0}:=\left\{z \in H^{2}\left([0,1], \mathbb{R}^{2}\right):\left|z^{\prime}\right| \equiv 1\right\}
$$

Theorem 2.1. Let $z_{I} \in \mathcal{A}_{0}$. Then there are $z_{0} \in H_{t, \mathrm{loc}}^{1} L_{s}^{2} \cap \mathcal{C}_{t}^{0, \frac{1}{2}} L_{s}^{2} \cap \mathcal{C}_{t}^{0, \frac{1}{8}} \mathcal{C}_{s}^{1} \cap L_{t}^{\infty} H_{s}^{2}$ and $\bar{\lambda}_{0} \in L_{\mathrm{loc}, t}^{2} \mathcal{M}_{s}$ such that the pair $\left(z_{0}, \bar{\lambda}_{0}\right)$ is a weak solution of (1.6) satisfying

$$
\int_{0}^{\infty} \int_{0}^{1}\left[z_{0}^{\prime \prime} \cdot v^{\prime \prime}+\partial_{t} z_{0} \cdot v+\bar{\lambda}_{0} z_{0}^{\prime} \cdot v^{\prime}\right] d s d t=0
$$

for all $v \in \mathcal{C}_{c}^{\infty}\left(\mathbb{R}_{+}, \mathcal{C}_{s}^{\infty}\right), z_{0}(t=0,)=.z_{0, I}$ and the constraint (1.7) in a pointwise sense for all $t \geq 0$ and $s \in[0,1]$.

THEOREM 2.2. The solution according to Theorem 2.1 gives a distributional sense to the system (1.18), i.e. for $\omega_{0}$ being the indicatrix of $z_{0}$ and $\lambda_{0}=\bar{\lambda}_{0}+\omega^{\prime 2}$ it holds that

$$
\begin{aligned}
& \int_{0}^{\infty} \int_{0}^{1}\left[-\omega_{0} \partial_{t} \psi-\omega_{0}^{\prime \prime \prime} \psi^{\prime}-\omega_{0}^{\prime \prime}\left(\omega_{0}^{\prime}\right)^{2} \psi-\lambda_{0}^{\prime} \omega_{0}^{\prime} \psi+\lambda_{0} \omega_{0}^{\prime} \psi^{\prime}\right] d s d t=0 \quad \text { and } \\
& \int_{0}^{\infty} \int_{0}^{1}\left[-\omega_{0}^{\prime \prime \prime} \omega_{0}^{\prime} \phi+\omega_{0}^{\prime \prime} \omega_{0}^{\prime} \phi^{\prime}+\lambda_{0}^{\prime} \phi^{\prime}+\lambda_{0}\left(\omega_{0}^{\prime}\right)^{2} \phi\right] d s d t=0
\end{aligned}
$$

for all $\psi \in H_{0, t}^{1} L_{s}^{2} \cap L_{t}^{2} H_{0, s}^{1}, \phi \in L_{t}^{2} H_{0, s}^{1}$, and $\lambda_{0}, \omega_{0}^{\prime},\left.\omega_{0}^{\prime \prime}\right|_{0,1}=0$ a.e. on $\mathbb{R}_{+}$. Furthermore it holds that $\omega_{0} \in \mathcal{C}_{t}^{0,1 / 8} \mathcal{C}_{s}^{0}$ with $\omega_{0}^{\prime} \in L_{t}^{\infty} L_{s}^{2} \cap L_{t}^{2} H_{s}^{2}$ and $\lambda_{0} \in L_{t}^{2} H_{s}^{1}$.

THEOREM 2.3. Let $z_{I} \in \mathcal{A}_{0}$, let $\left(z_{0}, \bar{\lambda}_{0}\right)$ be a solution of problem (1.6) according to Theorem 2.1, and let $\left(\omega_{0}, \lambda_{0}\right)$ be the corresponding solution to (1.18) according to Theorem 2.2. The curvature energy can then be equivalently formulated in terms of $z_{0}$ and in terms of $\omega_{0}$ as

$$
\mathcal{E}_{0}:=\int_{0}^{1} \frac{1}{2}\left|z_{0}^{\prime \prime}\right|^{2} d s=\int_{0}^{1} \frac{1}{2}\left(\omega_{0}^{\prime}\right)^{2} d s,
$$

and the curvature energy of the initial datum is given by

$$
\mathcal{E}_{0, I}:=\int_{0}^{1} \frac{1}{2}\left|z_{0, I}^{\prime \prime}\right|^{2} d s
$$


The energy dissipation is then given by

$$
\frac{d}{d t} \mathcal{E}_{0}=-\mathcal{D}_{0} \quad \text { with } \quad \mathcal{D}_{0}:=\int_{0}^{1}\left[\left(\omega_{0}^{\prime} \omega_{0}^{\prime \prime}+\lambda_{0}^{\prime}\right)^{2}+\left(\omega_{0}^{\prime \prime \prime}-\omega_{0}^{\prime} \lambda_{0}\right)^{2}\right] d s,
$$

in the sense that (2.6) holds weakly in time.

Finally the coercivity of $\mathcal{D}_{0}$ with respect to the curvature energy $\mathcal{E}_{0}$ (a Poincaré type inequality) yields the exponential decay of the energy.

Theorem 2.4. (Poincaré type inequality) Under the assumptions of Theorem 2.3, let the energy of the initial datum be given by (2.5), then it holds that

$$
\mathcal{E}_{0} \leq \mathcal{E}_{0, I} \exp \left(-2 \pi^{4} t\right)
$$

where again the curvature energy is alternatively given by (2.4).

As a consequence it holds that

$$
\left\|\partial_{t} z_{0}\right\|_{L_{t}^{2} L_{s}^{2}}^{2}=\int_{0}^{\infty} \mathcal{D}_{0} d t=\mathcal{E}_{0, I} .
$$

Finally we add here a technical statement which derives from the definition of the integrand of the punishing potential (1.4) two inequalities that we will use below in Section 6 and Section 7.

Lemma 2.5. Let the punishing profile be as defined in (1.4), which implies among other things that $E^{\prime \prime}(x) \geq \kappa$ for all $x \in\left(b_{\min }, b_{\max }\right)$. Then it satisfies

$$
\frac{\kappa}{2}(x-1)^{2} \leq E(x) \leq \frac{1}{2 \kappa} E^{\prime}(x)^{2}
$$

for all $x \in\left(b_{\min }, b_{\max }\right)$.

Proof. If $1 \leq x<b_{\max }$ integrate $E^{\prime \prime}(x) \geq \kappa$ twice on $(1, x)$ to obtain the first inequality. For the second inequality use $E^{\prime}(x) \geq 0$ to obtain $E^{\prime}(x) E^{\prime \prime}(x) \geq \kappa E^{\prime}(x)$ which gives the result after one integration.

If $b_{\min }<x<1$ integrate instead on $(x, 1)$ and use $-E^{\prime}(x) \geq 0$.

\section{Existence of solutions}

The definition of the curvature energy (1.2) and the notation used in (1.10) imply that

$$
\int_{0}^{1}\left(\omega^{\prime}\right)^{2} d s \leq 2 b_{\min }^{-2} \mathcal{E}^{\mathrm{curv}}[z], \quad \int_{0}^{1}\left(b^{\prime}\right)^{2} d s \leq 2 \mathcal{E}^{\mathrm{curv}}[z], \quad \text { and } \quad \int_{0}^{1}\left|z^{\prime \prime}\right|^{2} d s \leq 2 \mathcal{E}^{\mathrm{curv}}[z] .
$$

We introduce the time step approximation scheme

$$
Z_{\varepsilon}^{0}=z_{I} \quad \text { and } \quad Z_{\varepsilon}^{n} \in \operatorname{argmin}_{w:[0,1] \mapsto \mathbb{R}}\left\{\mathcal{E}_{\tau}\left(Z_{\varepsilon}^{n-1}\right)[w]+\mathcal{E}_{\varepsilon}[w]\right\},
$$

where $\tau>0$ is the constant size of the time steps, $n=0,1, \ldots$ is the index of the respective time step, and

$$
\mathcal{E}_{\tau}\left(Z_{\varepsilon}^{n-1}\right)[w]:=\int_{0}^{1} \frac{\left|w(s)-Z_{\varepsilon}^{n-1}\right|^{2}}{2 \tau} d s .
$$


Lemma 3.1. For any given $u \in \mathcal{A}$ there are constants $C_{1}>0$ and $C_{2}>0$ such that

$$
\mathcal{E}_{\tau}(u)[w]+\mathcal{E}_{\varepsilon}[w] \geq C_{1}\|w\|_{H_{s}^{2}}-C_{2} .
$$

Proof. Here we use (3.1) and the fact that the norm $\left|\|w \mid\|:=\|w\|_{L^{2}}+\left\|\partial_{s s} w\right\|_{L^{2}}\right.$ is equivalent to the usual norm in $H_{s}^{2}$.

Lemma 3.2. Let $\tau>0$ and $Z_{\varepsilon}^{n} \in \mathcal{A}$. Then $\mathcal{E}^{\text {curv }}$ is weakly lower semicontinuous and $\mathcal{E}_{\varepsilon}^{\text {pen }}$ and $\mathcal{E}_{\tau}$ are weakly continuous with respect to the $H^{2}(0,1)^{2}$-topology.

Proof. Weak lower semicontinuity is a consequence of the convexity of $\mathcal{E}^{\text {curv }}$. The integrand of $\mathcal{E}_{\tau}$ only depends on $w$ in a Lipschitz continuous way. Since due to Lemma 3.1 and embedding into $\mathcal{C}^{1}$ there is a $\delta>0$ such that $b_{\min }+\delta \leq\left|w^{\prime}\right| \leq b_{\max }-\delta$, the same is true for $\mathcal{E}_{\varepsilon}^{\text {pen }}$ with respect to $w^{\prime}$. The result is therefore a consequence of the compact embedding of $H^{2}(0,1)$ in $\mathcal{C}^{1}([0,1])$.

This proves the existence of a sequence $\left(Z_{\varepsilon}^{n}\right)_{n=0,1, \ldots}$ defined by $(3.2)$ with $Z_{\varepsilon}^{n} \in H_{s}^{2}$. Approximations of the solution of the continuous problem are then defined by linear interpolation and by piecewise constant extension:

$$
\left.\begin{array}{l}
Z_{\varepsilon, \tau}(t, s):=Z_{\varepsilon}^{n}(s)+\left(\frac{t}{\tau}-n\right)\left(Z_{\varepsilon}^{n+1}(s)-Z_{\varepsilon}^{n}(s)\right), \\
Z_{\varepsilon, \tau}^{\text {old }}(t, s):=Z_{\varepsilon}^{n}(s), \\
Z_{\varepsilon, \tau}^{\text {new }}(t, s):=Z_{\varepsilon}^{n+1}(s),
\end{array}\right\} \text { for } n \tau<t \leq(n+1) \tau .
$$

Lemma 3.3. For every fixed finite $T>0, Z_{\varepsilon, \tau} \in H^{1}\left((0, T), L^{2}(0,1)\right)$ uniformly in $\tau$.

Proof. The variational principle (3.2) implies that

$$
\int_{0}^{1}\left[\frac{1}{2 \tau}\left(Z_{\varepsilon}^{n}-Z_{\varepsilon}^{n-1}\right)^{2}\right] d s+\mathcal{E}_{\varepsilon}\left[Z_{\varepsilon}^{n}\right] \leq \mathcal{E}_{\varepsilon}\left[Z_{\varepsilon}^{n-1}\right]
$$

As a consequence it holds that

$$
\frac{1}{2 \tau}\left\|Z_{\varepsilon}^{n+1}-Z_{\varepsilon}^{n}\right\|_{L^{2}(0,1)}^{2} \leq \mathcal{E}_{\varepsilon}\left[Z^{n}\right]-\mathcal{E}_{\varepsilon}\left[Z^{n+1}\right] .
$$

Since the time derivative of $Z_{\varepsilon, \tau}$ is piecewise constant, taking the sum $n=0,1, \ldots, m-1$ in (3.4) where $m=\lceil T / \tau\rceil$ implies

$$
\int_{0}^{m \tau}\left\|\partial_{t} Z_{\varepsilon, \tau}\right\|_{L^{2}(0,1)}^{2} d t=\frac{1}{\tau} \sum_{n=0}^{m-1}\left\|Z_{\varepsilon}^{n+1}-Z_{\varepsilon}^{n}\right\|_{L^{2}(0,1)}^{2} \leq 2 \mathcal{E}_{\varepsilon}\left[z_{I}\right],
$$

completing the proof.

This result sets the stage for passing to the limit in the approximate solutions.

Lemma 3.4. For every fixed finite $T>0$,

$$
\lim _{\tau \rightarrow 0} Z_{\varepsilon, \tau}=z_{\varepsilon} \in L^{\infty}\left((0, T) ; H^{2}(0,1)\right) \cap \mathcal{C}^{0,1 / 8}\left([0, T] ; \mathcal{C}^{1}([0,1])\right) \cap H^{1}\left((0, T) ; L^{2}(0,1)\right),
$$

(restricting to subsequences) where the convergence is strong in $C\left([0, T] ; \mathcal{C}^{1}([0,1])\right)$, weak in $H^{1}\left((0, T) ; L^{2}(0,1)\right)$, and weak ${ }^{*}$ in $L^{\infty}\left((0, T) ; H^{2}(0,1)\right)$. The piecewise constant approximations $Z_{\varepsilon, \tau}^{\text {old }}$ and $Z_{\varepsilon, \tau}^{\text {new }}$ converge to $z$ strongly in $L^{\infty}\left([0, T] ; \mathcal{C}^{1}([0,1])\right)$ and weakly* in $L^{\infty}\left((0, T) ; H^{2}(0,1)\right)$. 
Proof. The inequalities (3.3) and (3.1) imply that $\left(Z_{\varepsilon, \tau}\right)_{\tau}$ is bounded in $L^{\infty}\left((0, T) ; H^{2}(0,1)\right)$ uniformly with respect to $\tau$, which already shows the weak* convergence. The weak convergence is a consequence of the previous lemma since the inequality (3.5) for $T \rightarrow \infty$ implies that

$$
\left\|\partial_{t} z_{\varepsilon}\right\|_{L_{t}^{2} L_{s}^{2}}^{2} \leq 2 \mathcal{E}_{\varepsilon}\left[z_{I}\right]
$$

Another consequence is that $Z_{\varepsilon, \tau}$ is uniformly bounded in $\mathcal{C}^{0,1 / 2}\left([0, T] ; L^{2}(0,1)\right)$. The interpolation inequality

$$
\|u\|_{\mathcal{C}^{1, \alpha}([0,1])} \leq c\|u\|_{L^{2}(0,1)}^{(1-2 \alpha) / 4}\|u\|_{H^{2}(0,1)}^{(3+2 \alpha) / 4}
$$

for $0 \leq \alpha \leq 1 / 2$, can then be used together with the $H^{2}(0,1)$-bound to obtain

$$
\left\|Z_{\varepsilon, \tau}\left(t_{2}\right)-Z_{\varepsilon, \tau}\left(t_{1}\right)\right\|_{\mathcal{C}^{1, \alpha}([0,1])} \leq c_{T}\left|t_{2}-t_{1}\right|^{(1-2 \alpha) / 8},
$$

completing the convergence proof for $Z_{\varepsilon, \tau}$ by an application of the Arzelà-Ascoli theorem. The convergence results for $Z_{\varepsilon, \tau}^{\text {old }}$ and $Z_{\varepsilon, \tau}^{\text {new }}$ are straightforward consequences.

This implies Theorem 1.1 due to the following argument.

Proof. (Proof of Theorem 1.1.) By construction the variational equation of $(3.2)$

$$
\delta \mathcal{E}_{\tau}\left(Z_{\varepsilon}^{n}\right)\left[Z_{\varepsilon}^{n+1}\right] v(t, \cdot)+\delta \mathcal{E}_{\varepsilon}\left(Z_{\varepsilon}^{n}\right)\left[Z_{\varepsilon}^{n+1}\right] v(t, \cdot)=0,
$$

holds for a test function $v \in \mathcal{C}_{c}^{\infty}\left(\mathbb{R}_{+}, \mathcal{C}_{s}^{\infty}\right)$ and $n \tau<t \leq(n+1) \tau$. With the definitions of $Z_{\varepsilon, \tau}, Z_{\varepsilon, \tau}^{\text {old }}$, and $Z_{\varepsilon, \tau}^{\text {new }}$ this can be written as

$$
\int_{0}^{1}\left[\partial_{t} Z_{\varepsilon, \tau} \cdot v+\partial_{s}^{2} Z_{\varepsilon, \tau}^{\text {new }} \cdot \partial_{s}^{2} v+\frac{1}{\varepsilon} E^{\prime}\left(\left|\partial_{s} Z_{\varepsilon, \tau}^{\text {new }}\right|\right) \frac{\partial_{s} Z_{\varepsilon, \tau}^{\text {new }}}{\left|\partial_{s} Z_{\varepsilon, \tau}^{\text {new }}\right|} \cdot \partial_{s} v\right] d s=0 .
$$

After integration with respect to $t$, we pass to the limit. Note that the weakly convergent terms $\partial_{s}^{2} Z_{\tau}^{\text {new }}$ and $\partial_{t} Z_{\varepsilon, \tau}$ occur only linearly, and that all other terms converge strongly.

\section{Indicatrix representation}

As mentioned above, $r_{\varepsilon}$ and $\lambda_{\varepsilon}$, which are defined in (1.12), are two components of $z_{\varepsilon}^{\prime \prime \prime}-\frac{1}{\varepsilon} E^{\prime}\left(\left|z_{\varepsilon}^{\prime}\right|\right) \frac{z^{\prime}}{\left|z^{\prime}\right|}$, namely

$$
\begin{aligned}
& r_{\varepsilon}=\left(z_{\varepsilon}^{\prime \prime \prime}-\frac{1}{\varepsilon} E^{\prime}\left(\left|z_{\varepsilon}^{\prime}\right|\right) \frac{z_{\varepsilon}^{\prime}}{\left|z_{\varepsilon}^{\prime}\right|}\right) \cdot \frac{z_{\varepsilon}^{\prime \perp}}{\left|z_{\varepsilon}^{\prime}\right|}=z_{\varepsilon}^{\prime \prime \prime} \cdot \frac{z_{\varepsilon}^{\prime \perp}}{\left|z_{\varepsilon}^{\prime}\right|} \\
& \lambda_{\varepsilon}=\left(z_{\varepsilon}^{\prime \prime \prime}-\frac{1}{\varepsilon} E^{\prime}\left(\left|z_{\varepsilon}^{\prime}\right|\right) \frac{z_{\varepsilon}^{\prime}}{\left|z_{\varepsilon}^{\prime}\right|}\right) \cdot \frac{z_{\varepsilon}^{\prime}}{\left|z_{\varepsilon}^{\prime}\right|}=z_{\varepsilon}^{\prime \prime \prime} \cdot \frac{z_{\varepsilon}^{\prime}}{\left|z_{\varepsilon}^{\prime}\right|}-\frac{1}{\varepsilon} E^{\prime}\left(\left|z_{\varepsilon}^{\prime}\right|\right) .
\end{aligned}
$$

Hence the system (1.5) can be written as

$$
\left\{\begin{array}{l}
\partial_{t} z_{\varepsilon}+\left(r_{\varepsilon} \frac{z_{\varepsilon}^{\prime \perp}}{\left|z_{\varepsilon}^{\prime}\right|}\right)^{\prime}-\left(\lambda_{\varepsilon} \frac{z_{\varepsilon}^{\prime}}{\left|z_{\varepsilon}^{\prime}\right|}\right)^{\prime}=0 \\
z_{\varepsilon}^{\prime \prime}, r_{\varepsilon},\left.\lambda_{\varepsilon}\right|_{s=0,1}=0 \\
z_{\varepsilon}(t=0, .)=z_{I}(.)
\end{array}\right.
$$


The definitions in (1.12) are motivated by the variation of $\mathcal{E}_{\varepsilon}$ as formulated in (1.10),

$$
\begin{aligned}
\delta \mathcal{E}_{\varepsilon}[\omega, b](\delta \omega, \delta b) & =\int_{0}^{1}\left(b^{2} \omega^{\prime}(\delta \omega)^{\prime}+b\left(\omega^{\prime}\right)^{2} \delta b+b^{\prime} \delta b^{\prime}+\frac{1}{\varepsilon} E^{\prime}(b) \delta b\right) d s \\
& =\left[b^{2} \omega^{\prime} \delta \omega+b^{\prime} \delta b\right]_{0}^{1}+\int_{0}^{1}-\left(b^{2} \omega^{\prime}\right)^{\prime} \delta \omega+\left(b\left(\omega^{\prime}\right)^{2}-b^{\prime \prime}+\frac{1}{\varepsilon} E^{\prime}(b)\right) \delta b d s .
\end{aligned}
$$

Replacing the variations of the indicatrix and of the arc-length by $\delta \omega=z^{\perp} \cdot \delta z^{\prime} /\left|z^{\prime}\right|^{2}$ and $\delta b=z^{\prime} \cdot \delta z^{\prime} /\left|z^{\prime}\right|$, respectively, we obtain

$$
\begin{aligned}
& \delta \mathcal{E}_{\varepsilon} \delta z=\left[\left(b \omega^{\prime} \frac{z^{\prime \perp}}{\left|z^{\prime}\right|}+b^{\prime} \frac{z^{\prime}}{\left|z^{\prime}\right|}\right) \cdot \delta z^{\prime}\right]_{0}^{1} \\
&+\int_{0}^{1} \delta z^{\prime} \cdot\left(-\frac{1}{b}\left(b^{2} \omega^{\prime}\right)^{\prime} \frac{z^{\prime \perp}}{\left|z^{\prime}\right|}+\left(b\left(\omega^{\prime}\right)^{2}-b^{\prime \prime}+\frac{1}{\varepsilon} E^{\prime}(b)\right) \frac{z^{\prime}}{\left|z^{\prime}\right|}\right) d s
\end{aligned}
$$

which motivates the definitions (1.12). The boundary terms in (4.4) also suggest that we replace the boundary condition $\left.z_{\varepsilon}^{\prime \prime}\right|_{s=0,1}=0$ by $\omega_{\varepsilon}^{\prime},\left.b_{\varepsilon}^{\prime}\right|_{s=0,1}=0$.

Lemma 4.1. Let $z_{I} \in \mathcal{A}$ and let $z_{\varepsilon}$ be a weak solution to problem (1.5) according to Theorem 1.1. Then there is $\omega_{\varepsilon} \in \mathcal{C}_{t}^{0,1 / 8} \mathcal{C}_{s}^{0} \cap L_{t}^{\infty} H_{s}^{1}$ such that $\omega_{\varepsilon}$ is an indicatrix of $z_{\varepsilon}$. Furthermore it holds that $b_{\varepsilon} \in \mathcal{C}_{t}^{0,1 / 8} \mathcal{C}_{s}^{0} \cap L_{t}^{\infty} H_{s}^{1}$ and $\left(z_{\varepsilon}, \omega_{\varepsilon}, b_{\varepsilon}\right)$ constitute a weak solution of (4.3) satisfying

$$
\begin{aligned}
\int_{0}^{\infty} \int_{0}^{1}\left[\partial_{t} z_{\varepsilon} \cdot v+b_{\varepsilon}^{2} \omega_{\varepsilon}^{\prime}\right. & \left(\frac{1}{b_{\varepsilon}} \frac{z_{\varepsilon}^{\prime \perp}}{\left|z_{\varepsilon}^{\prime}\right|} \cdot v_{\varepsilon}^{\prime}\right)^{\prime} \\
& \left.+\left(b_{\varepsilon}\left(\omega_{\varepsilon}^{\prime}\right)^{2}+\frac{1}{\varepsilon} E^{\prime}\left(b_{\varepsilon}\right)\right) \frac{z_{\varepsilon}^{\prime}}{\left|z_{\varepsilon}^{\prime}\right|} \cdot v^{\prime}+b_{\varepsilon}^{\prime}\left(\frac{z_{\varepsilon}^{\prime}}{\left|z_{\varepsilon}^{\prime}\right|} \cdot v^{\prime}\right)^{\prime}\right] d s d t=0
\end{aligned}
$$

for all $v \in \mathcal{C}_{c}^{\infty}\left(\mathbb{R}_{+}, \mathcal{C}_{s}^{\infty}\right)$.

Proof. The regularity of $z_{\varepsilon}^{\prime}$ according to Theorem 1.1 allows us to identify $\omega_{\varepsilon} \in \mathcal{C}_{t}^{0,1 / 8} \mathcal{C}_{s}^{0} \cap L_{t}^{\infty} H_{s}^{1}$ up to an additive constant which is a multiple of $2 \pi$. The regularity of $b_{\varepsilon}$ is due to its definition and the result of Theorem 1.1. The rest of the statement is then a consequence of the discussion above.

Lemma 4.2 (Regularity, a-priori estimates). Let $\left(z_{\varepsilon}, \omega_{\varepsilon}, \lambda_{\varepsilon}\right)$ be a weak solution of (4.3) in the sense of Lemma 4.1, where we use the notation (1.12). Then it holds that $r_{\varepsilon} \in L_{t}^{2} L_{s}^{\infty}, \omega_{\varepsilon}^{\prime} r_{\varepsilon} \in L_{t}^{2} L_{s}^{2}, \lambda_{\varepsilon} \in L_{t}^{2} L_{s}^{\infty}, \omega_{\varepsilon}^{\prime} \lambda_{\varepsilon} \in L_{t}^{2} L_{s}^{2}, r_{\varepsilon}^{\prime} \in L_{t}^{2} L_{s}^{2}$, and $\lambda_{\varepsilon}^{\prime} \in L_{t}^{2} L_{s}^{2}$. It also holds that

$$
\int_{0}^{\infty} \int_{0}^{1}\left[\left(r_{\varepsilon}^{\prime}-\lambda_{\varepsilon} \omega_{\varepsilon}^{\prime}\right)^{2}+\left(\lambda_{\varepsilon}^{\prime}+\omega_{\varepsilon}^{\prime} r_{\varepsilon}\right)^{2}\right] d s d t=\left\|\partial_{t} z_{\varepsilon}\right\|_{L_{t}^{2} L_{s}^{2}}^{2}
$$

Proof. First note that $\int_{0}^{s} \partial_{t} z_{\varepsilon} \in L_{t}^{2} L_{s}^{\infty}$ since

$$
\int_{0}^{\infty}\left\|\int_{0}^{s} \partial_{t} z_{\varepsilon} d s\right\|_{\infty}^{2} d t \leq \int_{0}^{\infty}\left(\int_{0}^{1}\left|\partial_{t} z_{\varepsilon}\right| d s\right)^{2} d t \leq \int_{0}^{\infty} \int_{0}^{1}\left|\partial_{t} z_{\varepsilon}\right|^{2} d s d t<2 \mathcal{E}_{I}
$$

by (3.6). We go back to the integrated version of (4.3),

$$
\left\{\begin{array}{l}
\int_{0}^{s} \partial_{t} z_{\varepsilon} d s+r_{\varepsilon} \frac{z_{\varepsilon}^{\prime \perp}}{\left|z_{\varepsilon}^{\prime}\right|}-\lambda_{\varepsilon} \frac{z_{\varepsilon}^{\prime}}{\left|z_{\varepsilon}^{\prime}\right|}=0 \\
\omega_{\varepsilon}^{\prime},\left.b_{\varepsilon}^{\prime}\right|_{s=0,1}=0 \\
z_{\varepsilon}(t=0, .)=z_{I}(.)
\end{array}\right.
$$


where $\omega_{\varepsilon}^{\prime},\left.b_{\varepsilon}^{\prime}\right|_{s=0,1}=0$ is an equivalent notation for $\left.z_{\varepsilon}^{\prime \prime}\right|_{s=0,1}=0$, and obtain

$$
\frac{z_{\varepsilon}^{\prime \perp}}{\left|z_{\varepsilon}^{\prime}\right|} \cdot \int_{0}^{s} \partial_{t} z_{\varepsilon} d s=-r_{\varepsilon} \quad \text { and } \quad \frac{z_{\varepsilon}^{\prime}}{\left|z_{\varepsilon}^{\prime}\right|} \cdot \int_{0}^{s} \partial_{t} z_{\varepsilon} d s=\lambda_{\varepsilon},
$$

which implies $r_{\varepsilon} \in L_{t}^{2} L_{s}^{\infty}$ and $\lambda_{\varepsilon} \in L_{t}^{2} L_{s}^{\infty}$.

Moreover we find that

$$
\begin{aligned}
\int_{0}^{\infty} \int_{0}^{1}\left(\lambda_{\varepsilon} \omega_{\varepsilon}^{\prime}\right)^{2} d s d t & \leq \int_{0}^{\infty}\left\|\lambda_{\varepsilon}\right\|_{L_{s}^{\infty}}^{2} \int_{0}^{1}\left(\omega_{\varepsilon}^{\prime}\right)^{2} d s d t \\
& \leq 2 \mathcal{E}_{I} b_{\min }^{-2} \int_{0}^{\infty}\left\|\lambda_{\varepsilon}\right\|_{L_{s}^{\infty}}^{2} d t<\left(2 \mathcal{E}_{I}\right)^{2} b_{\min }^{-2}
\end{aligned}
$$

where we used (3.1). This computation and an analogous one for $\omega_{\varepsilon}^{\prime} r_{\varepsilon}$ imply

$$
\omega_{\varepsilon}^{\prime} \lambda_{\varepsilon} \in L_{t}^{2} L_{s}^{2} \quad \text { and } \quad \omega_{\varepsilon}^{\prime} r_{\varepsilon} \in L_{t}^{2} L_{s}^{2}
$$

We write the weak formulation (4.5) of problem (4.3) after two integrations by parts,

$$
\begin{aligned}
\int_{0}^{\infty} \int_{0}^{1}\left(\partial_{t} z_{\varepsilon}\right. & \left.+\left(r_{\varepsilon} \frac{z_{\varepsilon}^{\prime \perp}}{\left|z_{\varepsilon}^{\prime}\right|}\right)^{\prime}-\left(\lambda_{\varepsilon} \frac{z_{\varepsilon}^{\prime}}{\left|z_{\varepsilon}^{\prime}\right|}\right)^{\prime}\right) \cdot v d s d t \\
& +\int_{0}^{\infty}\left[-r_{\varepsilon} \frac{z_{\varepsilon}^{\prime \perp}}{\left|z_{\varepsilon}^{\prime}\right|} \cdot v+\lambda_{\varepsilon} \frac{z_{\varepsilon}^{\prime}}{\left|z_{\varepsilon}^{\prime}\right|} \cdot v+b_{\varepsilon} \omega_{\varepsilon}^{\prime} \frac{z_{\varepsilon}^{\prime \perp}}{\left|z_{\varepsilon}^{\prime}\right|} \cdot v^{\prime}+b_{\varepsilon}^{\prime} \frac{z_{\varepsilon}^{\prime}}{\left|z_{\varepsilon}^{\prime}\right|} \cdot v^{\prime}\right]_{0}^{1} d t=0
\end{aligned}
$$

for all $v \in \mathcal{C}_{c}^{\infty}\left(\mathbb{R}_{+}, \mathcal{C}_{s}^{\infty}\right)$.

The uniform estimates we obtained allow us to set $v=\frac{z_{\varepsilon}^{\prime \perp}}{\left|z_{\varepsilon}^{\prime}\right|} \phi$ for a test function $\phi \in \mathcal{D}\left(([0, \infty) \times[0,1])\right.$ and to obtain $\int_{0}^{\infty} \int_{0}^{1}\left[\partial_{t} z_{\varepsilon} \cdot \frac{z_{\varepsilon}^{\prime \perp}}{\left|z_{\varepsilon}^{\prime}\right|} \phi+r_{\varepsilon}^{\prime} \phi-\lambda_{\varepsilon} \omega_{\varepsilon}^{\prime} \phi\right] d s d t=0$.

Specializing (4.8) for $v=\frac{z_{\varepsilon}^{\prime}}{\left|z_{\varepsilon}^{\prime}\right|} \psi$ with $\psi \in \mathcal{D}([0, \infty) \times[0,1])$, we conclude by an analogous computation $\int_{0}^{\infty} \int_{0}^{1}\left[\partial_{t} z_{\varepsilon} \cdot \frac{z_{\varepsilon}^{\prime}}{\left|z_{\varepsilon}^{\prime}\right|} \psi-\omega_{\varepsilon}^{\prime} r_{\varepsilon} \psi-\lambda_{\varepsilon}^{\prime} \psi\right] d s d t=0$. Due to (3.6) and (4.7), this implies

$$
\frac{z_{\varepsilon}^{\prime \perp}}{\left|z_{\varepsilon}^{\prime}\right|} \cdot \partial_{t} z_{\varepsilon}+r_{\varepsilon}^{\prime}-\lambda_{\varepsilon} \omega_{\varepsilon}^{\prime}=0 \quad \text { a.e. } \quad \text { and } \quad \frac{z_{\varepsilon}^{\prime}}{\left|z_{\varepsilon}^{\prime}\right|} \cdot \partial_{t} z_{\varepsilon}-\omega_{\varepsilon}^{\prime} r_{\varepsilon}-\lambda_{\varepsilon}^{\prime}=0 \quad \text { a.e. }
$$

hence $r_{\varepsilon}^{\prime} \in L_{t}^{2} L_{s}^{2}$ and $\lambda_{\varepsilon}^{\prime} \in L_{t}^{2} L_{s}^{2}$. Finally (4.9) implies

$$
\begin{gathered}
\int_{0}^{\infty} \int_{0}^{1}\left[\left(r_{\varepsilon}^{\prime}-\lambda_{\varepsilon} \omega_{\varepsilon}^{\prime}\right)^{2}+\left(\lambda_{\varepsilon}^{\prime}+\omega_{\varepsilon}^{\prime} r_{\varepsilon}\right)^{2}\right] d s d t \\
=\int_{0}^{\infty} \int_{0}^{1}\left[\left(\frac{z_{\varepsilon}^{\prime \perp}}{\left|z_{\varepsilon}^{\prime}\right|} \cdot \partial_{t} z_{\varepsilon}\right)^{2}+\left(\frac{z_{\varepsilon}^{\prime}}{\left|z_{\varepsilon}^{\prime}\right|} \cdot \partial_{t} z_{\varepsilon}\right)^{2}\right] d s d t=\left\|\partial_{t} z_{\varepsilon}\right\|_{L_{t}^{2} L_{s}^{2}}^{2} .
\end{gathered}
$$
$b_{\varepsilon}$.

Using this result we also get insight into regularity of higher derivatives of $\omega_{\varepsilon}$ and

LEMma 4.3 (Regularity of higher derivatives). Let $\left(z_{\varepsilon}, \omega_{\varepsilon}, \lambda_{\varepsilon}\right)$ be a weak solution of (4.3) in the sense of Lemma 4.1. Then it holds for every $T>0$ that the family 
$\left\{\omega_{\varepsilon}\right\}$ is uniformly bounded in $L^{1}\left(0, T ; W^{3,1}(0,1)\right)$ and that the family $\left\{b_{\varepsilon}\right\}$ is uniformly bounded in $L^{1}\left(0, T ; W^{2,1}(0,1)\right)$. It also holds that $b_{\varepsilon}^{\prime \prime \prime} \in L^{2}\left(0, T ; L_{s}^{1}\right)$, but not uniformly with respect to $\varepsilon$. hence

Proof. For the proof we use the definitions in (1.12). It holds that $\omega_{\varepsilon}^{\prime \prime}=\frac{r_{\varepsilon}-2 b_{\varepsilon}^{\prime} \omega_{\varepsilon}^{\prime}}{b_{\varepsilon}}$,

$$
\left\|\omega_{\varepsilon}^{\prime \prime}\right\|_{L^{2}\left(0, T ; L_{s}^{1}\right)} \leq \frac{1}{b_{\min }}\left(\left\|r_{\varepsilon}\right\|_{L^{2}\left(0, T ; L_{s}^{2}\right)}+2\left\|b_{\varepsilon}^{\prime}\right\|_{L^{2}\left(0, T ; L_{s}^{2}\right)}\left\|\omega_{\varepsilon}^{\prime}\right\|_{L^{2}\left(0, T ; L_{s}^{2}\right)}\right),
$$

which is uniformly bounded due to Lemma 4.2 and Lemma 4.1.

Furthermore the definition of $r_{\varepsilon}$ in (1.12) also implies that $\omega_{\varepsilon}^{\prime \prime \prime}=r_{\varepsilon}^{\prime}-2 b_{\varepsilon}^{\prime \prime} \omega_{\varepsilon}^{\prime}-$ $\left.3 b_{\varepsilon}^{\prime} \omega_{\varepsilon}^{\prime \prime}\right) / b_{\varepsilon}$. Hence we estimate

$$
\begin{gathered}
\left\|\omega_{\varepsilon}^{\prime \prime \prime}\right\|_{L^{1}\left(0, T ; L_{s}^{1}\right)} \leq b_{\min }^{-1}\left(\left\|r_{\varepsilon}^{\prime}\right\|_{L^{1}\left(0, T ; L_{s}^{1}\right)}+2\left\|b_{\varepsilon}^{\prime \prime}\right\|_{L^{2}\left(0, T ; L_{s}^{1}\right)}\left\|\omega_{\varepsilon}^{\prime}\right\|_{L^{2}\left(0, T ; L_{s}^{\infty}\right)}\right. \\
\left.+3\left\|b_{\varepsilon}^{\prime}\right\|_{L^{2}\left(0, T ; L_{s}^{\infty}\right)}\left\|\omega_{\varepsilon}^{\prime \prime}\right\|_{L^{2}\left(0, T ; L_{s}^{1}\right)}\right) \\
\leq b_{\min }^{-1}\left(\sqrt{T}\left\|r_{\varepsilon}^{\prime}\right\|_{L^{2}\left(0, T ; L_{s}^{2}\right)}+5\left\|b_{\varepsilon}^{\prime \prime}\right\|_{L^{2}\left(0, T ; L_{s}^{1}\right)}\left\|\omega_{\varepsilon}^{\prime \prime}\right\|_{L^{2}\left(0, T ; L_{s}^{1}\right)}\right)
\end{gathered}
$$

which is uniformly bounded due to the result above.

Observe that due to Lemma 3.1 and embedding into $\mathcal{C}^{1}$ there is a $\delta>0$ such that $b_{\min }+\delta \leq\left|z_{\varepsilon}^{\prime}\right| \leq b_{\max }-\delta$. Hence, using $b_{\varepsilon}^{\prime \prime}=b_{\varepsilon}\left(\omega_{\varepsilon}^{\prime}\right)^{2}-\lambda_{\varepsilon}+\frac{1}{\varepsilon} E^{\prime}\left(b_{\varepsilon}\right)$, we conclude that

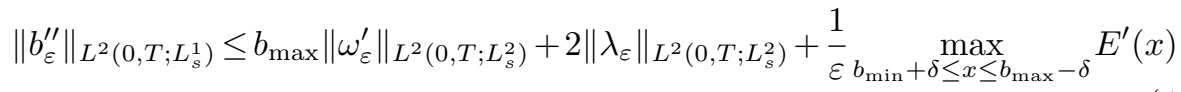

is bounded due to Lemma 4.2 and Lemma 4.1. This bound is not uniform with respect to $\varepsilon$, but can be replaced by a uniform one using the following computation.

Integrating the definition of $\lambda_{\varepsilon}$ against $E^{\prime}\left(b_{\varepsilon}\right)$ we obtain

$$
\int_{0}^{1} \lambda_{\varepsilon} E^{\prime}\left(b_{\varepsilon}\right) d \tilde{s}=\int_{0}^{1}\left[b_{\varepsilon}\left(\omega_{\varepsilon}^{\prime}\right)^{2} E^{\prime}\left(b_{\varepsilon}\right)+b_{\varepsilon}^{\prime 2} E^{\prime \prime}\left(b_{\varepsilon}\right)+\frac{1}{\varepsilon} E^{\prime}\left(b_{\varepsilon}\right)^{2}\right] d \tilde{s} .
$$

This implies the inequality

$$
\int_{0}^{1} \frac{1}{\varepsilon} E^{\prime}\left(b_{\varepsilon}\right)^{2} d \tilde{s} \leq \int_{0}^{1}\left(\lambda_{\varepsilon} E^{\prime}\left(b_{\varepsilon}\right)-b_{\varepsilon}\left(\omega_{\varepsilon}^{\prime}\right)^{2} E^{\prime}\left(b_{\varepsilon}\right)\right) d \tilde{s} \leq\left\|\lambda_{\varepsilon}-b_{\varepsilon}\left(\omega_{\varepsilon}^{\prime}\right)^{2}\right\|_{L^{2}}\left\|E^{\prime}\left(b_{\varepsilon}\right)\right\|_{L^{2}} .
$$

Hence we conclude that

$$
\frac{1}{\varepsilon}\left\|E^{\prime}\left(b_{\varepsilon}\right)\right\|_{L^{2}} \leq\left\|\lambda_{\varepsilon}-b_{\varepsilon}\left(\omega_{\varepsilon}^{\prime}\right)^{2}\right\|_{L^{2}},
$$

which allows us to replace (4.11) by

$$
\left\|b_{\varepsilon}^{\prime \prime}\right\|_{L^{2}\left(0, T ; L_{s}^{1}\right)} \leq b_{\max }\left\|\omega_{\varepsilon}^{\prime}\right\|_{L^{2}\left(0, T ; L_{s}^{2}\right)}+2\left\|\lambda_{\varepsilon}\right\|_{L^{2}\left(0, T ; L_{s}^{2}\right)}+\left\|\lambda_{\varepsilon}-b_{\varepsilon}\left(\omega_{\varepsilon}^{\prime}\right)^{2}\right\|_{L^{2}\left(0, T ; L_{s}^{2}\right)},
$$

which is uniformly bounded due to the results of Lemma 4.2 and Lemma 4.1.

Furthermore the definition of $\lambda_{\varepsilon}$ in (1.12) also implies that $b_{\varepsilon}^{\prime \prime \prime}=b_{\varepsilon}^{\prime}\left(\omega_{\varepsilon}^{\prime}\right)^{2}+$ $2 b_{\varepsilon} \omega_{\varepsilon}^{\prime} \omega_{\varepsilon}^{\prime \prime}-\lambda_{\varepsilon}^{\prime}+\frac{1}{\varepsilon} E^{\prime \prime}\left(b_{\varepsilon}\right) b_{\varepsilon}^{\prime}$. Hence we estimate

$$
\left\|b_{\varepsilon}^{\prime \prime \prime}\right\|_{L^{1}\left(0, T ; L_{s}^{1}\right)} \leq\left\|b_{\varepsilon}^{\prime}\right\|_{L^{1}\left(0, T ; L_{s}^{\infty}\right)}\left\|\left(\omega_{\varepsilon}^{\prime}\right)^{2}\right\|_{L^{\infty}\left(0, T ; L_{s}^{1}\right)}+2 b_{\max }\left\|\omega_{\varepsilon}^{\prime \prime}\right\|_{L^{2}\left(0, T ; L_{s}^{1}\right)}^{2}
$$




$$
+\left\|\lambda_{\varepsilon}^{\prime}\right\|_{L^{1}\left(0, T ; L_{s}^{2}\right)}+\frac{1}{\varepsilon}\left\|b_{\varepsilon}^{\prime}\right\|_{L^{1}\left(0, T ; L_{s}^{1}\right)} \max _{b_{\min }+\delta \leq x \leq b_{\max }-\delta} E^{\prime \prime}(x),
$$

which is bounded due to the result above, but not uniformly with respect to $\varepsilon$.

Next we will derive the governing equations for the quantities $\omega_{\varepsilon}$ and $b_{\varepsilon}$ making use of the following identities:

$$
\left(\frac{z^{\prime}}{\left|z^{\prime}\right|}\right)^{\prime}=\omega^{\prime} \frac{z^{\prime \perp}}{\left|z^{\prime}\right|} \text { and }\left(\frac{z^{\prime \perp}}{\left|z^{\prime}\right|}\right)^{\prime}=-\omega^{\prime} \frac{z^{\prime}}{\left|z^{\prime}\right|},
$$

which hold for a curve $z$ with sufficient regularity and its indicatrix $\omega$. We take formally the derivative of the evolution equation in (4.3) and evaluate explicitly all of the derivatives, obtaining

$$
\begin{aligned}
\partial_{t} z_{\varepsilon}^{\prime}+\left(r_{\varepsilon}^{\prime \prime}-\left(\lambda_{\varepsilon} \omega_{\varepsilon}^{\prime}\right)^{\prime}\right) \frac{z_{\varepsilon}^{\prime \perp}}{\left|z_{\varepsilon}^{\prime}\right|}-\left(r_{\varepsilon}^{\prime} \omega_{\varepsilon}^{\prime}-\lambda_{\varepsilon} \omega_{\varepsilon}^{\prime 2}\right) \frac{z_{\varepsilon}^{\prime}}{\left|z_{\varepsilon}^{\prime}\right|} & \\
& -\left(\lambda_{\varepsilon}^{\prime \prime}+\left(r_{\varepsilon}^{\prime} \omega_{\varepsilon}^{\prime}\right)^{\prime}\right) \frac{z_{\varepsilon}^{\prime}}{\left|z_{\varepsilon}^{\prime}\right|}-\left(\lambda_{\varepsilon}^{\prime \prime} \omega_{\varepsilon}^{\prime}+r_{\varepsilon}^{\prime} \omega_{\varepsilon}^{\prime 2}\right) \frac{z_{\varepsilon}^{\prime \perp}}{\left|z_{\varepsilon}^{\prime}\right|}=0 .
\end{aligned}
$$

We multiply by $z_{\varepsilon}^{\prime \perp} /\left|z_{\varepsilon}^{\prime}\right|^{2}$ and $z_{\varepsilon}^{\prime} /\left|z_{\varepsilon}^{\prime}\right|$ and use $\partial_{t} \omega_{\varepsilon}=\partial_{t} z_{\varepsilon}^{\prime} \cdot z_{\varepsilon}^{\prime \perp} /\left|z_{\varepsilon}^{\prime}\right|^{2}$ and $\partial_{t} b_{\varepsilon}=\partial_{t} z_{\varepsilon}^{\prime}$. $z_{\varepsilon}^{\prime} /\left|z_{\varepsilon}^{\prime}\right|$ to obtain (1.13).

We present the proof of Theorem 1.2, which collects the regularity statements of lemmas 4.1, 4.2, and 4.3 and justifies the above computation in the distributional sense.

Proof. (Theorem 1.2.) We start with a weak solution of (4.3) satisfying (4.5) and (4.8) respectively for all $v \in \mathcal{C}_{c}^{\infty}\left(\mathbb{R}_{+}, \mathcal{C}_{s}^{\infty}\right)$ according to Lemma 4.1. The boundary integrals imply that $\omega_{\varepsilon}^{\prime}, b_{\varepsilon}^{\prime}, \lambda_{\varepsilon},\left.r_{\varepsilon}\right|_{0,1}=0$ a.e. on $\mathbb{R}_{+}$.

We choose a regularizing sequence $\eta_{k}$ with $\operatorname{supp} \eta_{k} \subset B(0,1 / k) \subset \mathbb{R}^{2}$ and set $v=$ $\eta_{k}(\tilde{t}-t, \tilde{s}-s)$ for $(\tilde{t}, \tilde{s}) \in U_{k}$ with $U_{k}:=[1 / k, \infty) \times(1 / k, 1-1 / k)$, obtaining

$$
\partial_{t} z_{\varepsilon, k}+\left(r_{\varepsilon} \frac{z_{\varepsilon}^{\prime \perp}}{\left|z_{\varepsilon}^{\prime}\right|}\right)^{\prime} * \eta_{k}-\left(\lambda_{\varepsilon} \frac{z_{\varepsilon}^{\prime}}{\left|z_{\varepsilon}^{\prime}\right|}\right)^{\prime} * \eta_{k}=0
$$

where $z_{\varepsilon, k}:=z_{\varepsilon} * \eta_{k}$. We omit the tilde and integrate against $-\left(\frac{z_{\varepsilon, k}^{\prime}}{\left|z_{\varepsilon, k}^{\prime}\right|} \varphi+\frac{z_{\varepsilon, k}^{\prime \perp}}{\left|z_{\varepsilon, k}^{\prime}\right|^{2}} \psi\right)^{\prime}$ with $\varphi, \psi \in \mathcal{D}\left(\mathbb{R}_{+} \times[0,1]\right)$ and $k$ large enough such that $U_{k}$ covers the support of both test functions,

$$
\begin{aligned}
\iint_{U_{k}}\left[-\partial_{t} z_{\varepsilon, k}\right. & \cdot\left(z_{\varepsilon, k}^{\prime \perp} \frac{1}{\left|z_{\varepsilon, k}^{\prime}\right|^{2}} \psi\right)^{\prime} \\
& \left.\left(\left(r_{\varepsilon} \frac{z_{\varepsilon}^{\prime \perp}}{\left|z_{\varepsilon}^{\prime}\right|}\right)^{\prime} * \eta_{k}-\left(\lambda_{\varepsilon} \frac{z_{\varepsilon}^{\prime}}{\left|z_{\varepsilon}^{\prime}\right|}\right)^{\prime} * \eta_{k}\right) \cdot\left(z_{\varepsilon, k}^{\prime \perp} \frac{1}{\left|z_{\varepsilon, k}^{\prime}\right|^{2}} \psi\right)^{\prime}\right] d s d t=0, \\
\iint_{U_{k}}-\partial_{t} z_{\varepsilon, k} & \cdot\left(\frac{z_{\varepsilon, k}^{\prime}}{\left|z_{\varepsilon, k}^{\prime}\right|} \varphi\right)^{\prime}-\left(\left(r_{\varepsilon} \frac{z_{\varepsilon}^{\prime \perp}}{\left|z_{\varepsilon}^{\prime}\right|}\right)^{\prime} * \eta_{k}-\left(\lambda_{\varepsilon} \frac{z_{\varepsilon}^{\prime}}{\left|z_{\varepsilon}^{\prime}\right|}\right)^{\prime} * \eta_{k}\right) \cdot\left(\frac{z_{\varepsilon, k}^{\prime}}{\left|z_{\varepsilon, k}^{\prime}\right|} \varphi\right)^{\prime} d s d t=0 .
\end{aligned}
$$

Making use of the regularity results in Theorem 1.1 it holds that $z_{\varepsilon, k}^{\prime} \rightarrow z_{\varepsilon}^{\prime}$ and, as a consequence, also $\omega_{\varepsilon, k} \rightarrow \omega_{\varepsilon}$ uniformly on compact subsets of $\mathbb{R}_{+} \times[0,1]$, where $\omega_{\varepsilon, k}$ is 
the indicatrix of $z_{\varepsilon, k}$ satisfying $z_{\varepsilon, k}^{\prime} /\left|z_{\varepsilon, k}^{\prime}\right|=\left(\cos \left(\omega_{\varepsilon, k}\right), \sin \left(\omega_{\varepsilon, k}\right)\right)$. Using these results we perform integrations by parts with respect to $s$ and $t$ with the expressions in (4.15) that involve $\partial_{t} z_{\varepsilon, k}$ and pass to the limit, obtaining

$$
\begin{aligned}
\iint_{U_{k}}-\partial_{t} z_{\varepsilon, k} \cdot\left(\frac{z_{\varepsilon}^{\prime}}{\left|z_{\varepsilon}^{\prime}\right|} \varphi\right)^{\prime} d s d t & =\iint_{U_{k}} \partial_{t} z_{\varepsilon, k}^{\prime} \cdot \frac{z_{\varepsilon}^{\prime}}{\left|z_{\varepsilon}^{\prime}\right|} \varphi d s d t \\
& =\iint_{U_{k}}-\left|z_{\varepsilon, k}^{\prime}\right| \partial_{t} \varphi d s d t \rightarrow \iint_{U_{k}}-b_{\varepsilon} \partial_{t} \varphi d s d t \quad \text { as } \quad k \rightarrow \infty,
\end{aligned}
$$

and

$$
\begin{aligned}
\iint_{U_{k}}-\partial_{t} z_{\varepsilon, k} \cdot\left(z_{\varepsilon, k}^{\prime \perp} \frac{1}{\left|z_{\varepsilon, k}^{\prime}\right|^{2}} \psi\right)^{\prime} d s d t & =\iint_{U_{k}} \partial_{t} z_{\varepsilon, k}^{\prime} \cdot z_{\varepsilon, k}^{\prime \perp} \frac{1}{\left|z_{\varepsilon, k}^{\prime}\right|^{2}} \psi d s d t \\
& =\iint_{U_{k}} \partial_{t} \omega_{\varepsilon, k} \psi d s d t=\iint_{U_{k}}-\omega_{\varepsilon, k} \partial_{t} \psi d s d t \\
& \rightarrow \int_{0}^{\infty} \int_{0}^{1}-\omega_{\varepsilon} \partial_{t} \psi d s d t \quad \text { as } \quad k \rightarrow \infty,
\end{aligned}
$$

where we used that $\partial_{t} \omega_{\varepsilon, k}=\partial_{t} z_{\varepsilon, k}^{\prime} \cdot z_{\varepsilon, k}^{\prime \prime} \frac{1}{\left|z_{\varepsilon, k}^{\prime}\right|^{2}}$. Making use of the the fact that $z_{\varepsilon}^{\prime \prime} \in$ $L_{t}^{\infty} L_{s}^{2}$ by Theorem 1.1 and $r_{\varepsilon}^{\prime}, \lambda_{\varepsilon}^{\prime} \in L_{t}^{2} L_{s}^{2}$ by Lemma 4.2 , we now pass to the limit $k \rightarrow \infty$ in the remaining expressions of (4.15) and conclude that

$$
\begin{array}{r}
\int_{0}^{\infty} \int_{0}^{1}\left[-\omega_{\varepsilon} \partial_{t} \psi-\left(r_{\varepsilon}^{\prime} \frac{z_{\varepsilon}^{\prime \perp}}{\left|z_{\varepsilon}^{\prime}\right|}-\lambda_{\varepsilon}^{\prime} \frac{z_{\varepsilon}^{\prime}}{\left|z_{\varepsilon}^{\prime}\right|}-r_{\varepsilon} \omega_{\varepsilon}^{\prime} \frac{z_{\varepsilon}^{\prime}}{\left|z_{\varepsilon}^{\prime}\right|}-\lambda_{\varepsilon} \omega_{\varepsilon}^{\prime} \frac{z_{\varepsilon}^{\prime \perp}}{\left|z_{\varepsilon}^{\prime}\right|}\right)\right. \\
\left.\cdot\left(-\frac{z_{\varepsilon}^{\prime}}{\left|z_{\varepsilon}^{\prime}\right|} \omega_{\varepsilon}^{\prime} \frac{\psi}{\left|z_{\varepsilon}^{\prime}\right|}+\frac{z_{\varepsilon}^{\prime \perp}}{\left|z_{\varepsilon}^{\prime}\right|}\left(\frac{\psi}{\left|z_{\varepsilon}^{\prime}\right|}\right)^{\prime}\right)\right] d s d t=0, \\
\int_{0}^{\infty} \int_{0}^{1}\left[-b_{\varepsilon} \partial_{t} \varphi-\left(r_{\varepsilon}^{\prime} \frac{z_{\varepsilon}^{\prime \perp}}{\left|z_{\varepsilon}^{\prime}\right|}-\lambda_{\varepsilon}^{\prime} \frac{z_{\varepsilon}^{\prime}}{\left|z_{\varepsilon}^{\prime}\right|}-r_{\varepsilon} \omega_{\varepsilon}^{\prime} \frac{z_{\varepsilon}^{\prime}}{\left|z_{\varepsilon}^{\prime}\right|}-\lambda_{\varepsilon} \omega_{\varepsilon}^{\prime} \frac{z_{\varepsilon}^{\prime \perp}}{\left|z_{\varepsilon}^{\prime}\right|}\right)\right. \\
\left.\cdot\left(\frac{z_{\varepsilon}^{\prime \perp}}{\left|z_{\varepsilon}^{\prime}\right|} \omega_{\varepsilon}^{\prime} \varphi+\frac{z_{\varepsilon}^{\prime}}{\left|z_{\varepsilon}^{\prime}\right|} \varphi^{\prime}\right)\right] d s d t=0 .
\end{array}
$$

This implies $(1.20),(1.21)$ where every term is well defined by Lemma 4.2, and where we allow for $\psi, \phi$ in function spaces in which test functions are densely contained.

\section{Energy dissipation}

Observe that the formal time derivative of the total energy as formulated in (1.10) is given by

$$
\frac{d}{d t} \mathcal{E}_{\varepsilon}=\int_{0}^{1}\left(b^{2} \omega_{\varepsilon}^{\prime} \partial_{t} \omega_{\varepsilon}^{\prime}+\lambda_{\varepsilon} \partial_{t} b_{\varepsilon}\right) d s
$$

Now test the first equation in (1.13) formally against $\left(b_{\varepsilon}^{2} \omega_{\varepsilon}^{\prime}\right)^{\prime}=b_{\varepsilon} r_{\varepsilon}$, obtaining

$$
\int_{0}^{1} b^{2} \omega_{\varepsilon}^{\prime} \partial_{t} \omega_{\varepsilon}^{\prime} d s=\int_{0}^{1}\left[-\left(r_{\varepsilon}^{\prime}\right)^{2}-r_{\varepsilon}^{2}\left(\omega_{\varepsilon}^{\prime}\right)^{2}-\lambda_{\varepsilon}^{\prime} \omega_{\varepsilon}^{\prime} r_{\varepsilon}+\omega_{\varepsilon}^{\prime} \lambda_{\varepsilon} r_{\varepsilon}^{\prime}\right] d s
$$


and the second one in (1.13) against $\lambda_{\varepsilon}$, which yields

$$
\int_{0}^{1} \lambda_{\varepsilon} \partial_{t} b_{\varepsilon} d s=\int_{0}^{1}\left[r_{\varepsilon}^{\prime} \omega_{\varepsilon}^{\prime} \lambda_{\varepsilon}-\left(r_{\varepsilon} \omega_{\varepsilon}^{\prime}\right) \lambda_{\varepsilon}^{\prime}-\left(\lambda_{\varepsilon}^{\prime}\right)^{2}-\left(\omega_{\varepsilon}^{\prime}\right)^{2}\left(\lambda_{\varepsilon}\right)^{2}\right] d s .
$$

Take the sum to conclude the energy dissipation equality (1.14) on a formal level.

This can be made rigorous by stating that (1.14) holds weakly in time (Theorem 1.3).

Proof. (Theorem 1.3.) Here the problem is that we cannot directly set $\psi$ in (1.21) equal to $b_{\varepsilon} r_{\varepsilon}^{\prime}$, since its time derivative cannot necessarily be interpreted as a function. Therefore we regularize with respect to $t$ using a sequence of mollifiers $\left(\eta_{k}\right)_{k=1,2, \ldots}$ with $\operatorname{supp} \eta_{k} \subset[-1 / k, 1 / k]$.

For $\tilde{t} \geq 1 / k$ we denote by $\omega_{\varepsilon, k}(\tilde{t}, s):=\left(\omega_{\varepsilon} *_{t} \eta_{k}\right)(\tilde{t}, s)=\int_{0}^{\infty} \omega_{\varepsilon}(t, s) \eta_{k}(\tilde{t}-t) d t$ the regularized version of $\omega_{\varepsilon}$ and evaluate (1.21) using $\psi(t, s)=\eta_{k}(\tilde{t}-t)\left(b_{\varepsilon, k}^{2} \omega_{\varepsilon, k}^{\prime}\right)^{\prime}(\tilde{t}, s)$. The time integral becomes part of the convolution and we find the following expression, which holds pointwise for every $\tilde{t} \geq 1 / k$ :

$$
\begin{aligned}
\int_{0}^{1}\left[\partial_{t} \omega_{\varepsilon, k}\left(b_{\varepsilon, k}^{2} \omega_{\varepsilon, k}^{\prime}\right)^{\prime}-\left(\left(r_{\varepsilon}^{\prime}-\lambda_{\varepsilon} \omega_{\varepsilon}^{\prime}\right) \frac{1}{b_{\varepsilon}}\right) *_{t} \eta_{k}\left(b_{\varepsilon, k} r_{\varepsilon, k}\right)^{\prime}\right. \\
\left.\quad+\left(\left(r_{\varepsilon}^{\prime}-\lambda_{\varepsilon} \omega_{\varepsilon}^{\prime}\right) \frac{b_{\varepsilon}^{\prime}}{b_{\varepsilon}^{2}}\right) *_{t} \eta_{k} b_{\varepsilon, k} r_{\varepsilon, k}-\left(\left(r_{\varepsilon} \omega_{\varepsilon}^{\prime}+\lambda_{\varepsilon}^{\prime}\right) \frac{\omega_{\varepsilon}^{\prime}}{b_{\varepsilon}}\right) *_{t} \eta_{k} b_{\varepsilon, k} r_{\varepsilon, k}\right] d s=0 .
\end{aligned}
$$

Observe that we write the convolution of various expressions and $\eta_{k}$ explicitly using the symbol $*_{t}$. Furthermore, setting $\phi=\eta_{k}(\tilde{t}-t) \lambda_{\varepsilon, k}(\tilde{t}, s)$ in (1.20) where $\lambda_{\varepsilon, k}=$ $b_{\varepsilon, k}\left(\omega_{\varepsilon, k}^{\prime}\right)^{2}-b_{\varepsilon, k}^{\prime \prime}+\frac{1}{\varepsilon} E^{\prime}\left(b_{\varepsilon, k}\right)$,

$$
\int_{0}^{1}\left[\partial_{t} b_{\varepsilon, k} \lambda_{\varepsilon, k}+\left(r_{\varepsilon} \omega_{\varepsilon}^{\prime}+\lambda_{\varepsilon}^{\prime}\right) *_{t} \eta_{k} \lambda_{\varepsilon, k}^{\prime}+\left(\left(\omega_{\varepsilon}^{\prime} \lambda_{\varepsilon}-r_{\varepsilon}^{\prime}\right) \omega_{\varepsilon}^{\prime}\right) *_{t} \eta_{k} \lambda_{\varepsilon, k}\right] d s=0
$$

We omit the tildes, take the sum of the two equations above, and integrate against the test function in time $\vartheta \in \mathcal{D}\left(\mathbb{R}_{+}\right)$, obtaining

$$
\begin{aligned}
0=\int_{1 / k}^{\infty}\left[-\partial_{t} \vartheta\right. & \mathcal{E}_{\varepsilon, k}+\vartheta \int_{0}^{1}\left[\left(\left(r_{\varepsilon}^{\prime}-\lambda_{\varepsilon} \omega_{\varepsilon}^{\prime}\right) \frac{1}{b_{\varepsilon}}\right) *_{t} \eta_{k}\left(b_{\varepsilon, k} r_{\varepsilon, k}\right)^{\prime}\right. \\
& +\left(-\left(r_{\varepsilon}^{\prime}-\lambda_{\varepsilon} \omega_{\varepsilon}^{\prime}\right) \frac{b_{\varepsilon}^{\prime}}{b_{\varepsilon}^{2}}+\left(r_{\varepsilon} \omega_{\varepsilon}^{\prime}+\lambda_{\varepsilon}^{\prime}\right) \frac{\omega_{\varepsilon}^{\prime}}{b_{\varepsilon}}\right) *_{t} \eta_{k} b_{\varepsilon, k} r_{\varepsilon, k} \\
& \left.\left.+\left(r_{\varepsilon} \omega_{\varepsilon}^{\prime}+\lambda_{\varepsilon}^{\prime}\right) *_{t} \eta_{k} \lambda_{\varepsilon, k}^{\prime}+\left(\left(\omega_{\varepsilon}^{\prime} \lambda_{\varepsilon}-r_{\varepsilon}^{\prime}\right) \omega_{\varepsilon}^{\prime}\right) *_{t} \eta_{k} \lambda_{\varepsilon, k}\right] d s\right] d t \quad \forall \quad \tilde{t} \geq 1 / k
\end{aligned}
$$

where $\mathcal{E}_{\varepsilon, k}=\int_{0}^{1}\left[\frac{1}{2}\left(\omega_{\varepsilon, k}^{\prime 2} b_{\varepsilon, k}^{2}+\left(b_{\varepsilon, k}^{\prime}\right)^{2}\right)+\frac{1}{\varepsilon} E\left(b_{\varepsilon, k}\right)\right] d s$ and $k$ is large enough so that $\operatorname{supp} \vartheta \subset[1 / k, \infty)$. Next we use the fact that all the convolved terms like $\omega_{\varepsilon, k}^{\prime}, r_{\varepsilon, k}^{\prime}$, $\left(r_{\varepsilon}\left(\omega_{\varepsilon}^{\prime}\right)^{2}\right) *_{t} \eta_{k}$, etc. converge strongly in $L_{t, \text { loc }}^{2} L_{s}^{2}$ to their original counterparts, which is illustrated in more detail in the proof of Theorem 4 in [9]. Hence we pass to the limit as $k \rightarrow \infty$ in (5.1), obtaining

$$
\int_{0}^{\infty} \partial_{t} \vartheta \mathcal{E}_{\varepsilon} d t=\int_{0}^{\infty}\left[\vartheta \int _ { 0 } ^ { 1 } \left[\left(\left(r_{\varepsilon}^{\prime}-\lambda_{\varepsilon} \omega_{\varepsilon}^{\prime}\right) \frac{1}{b_{\varepsilon}}\right)\left(b_{\varepsilon} r_{\varepsilon}\right)^{\prime}\right.\right.
$$




$$
\begin{aligned}
&+\left(-\left(r_{\varepsilon}^{\prime}-\lambda_{\varepsilon} \omega_{\varepsilon}^{\prime}\right) \frac{b_{\varepsilon}^{\prime}}{b_{\varepsilon}^{2}}+\left(r_{\varepsilon} \omega_{\varepsilon}^{\prime}+\lambda_{\varepsilon}^{\prime}\right) \frac{\omega_{\varepsilon}^{\prime}}{b_{\varepsilon}}\right) b_{\varepsilon} r_{\varepsilon} \\
&\left.\left.+\left(r_{\varepsilon} \omega_{\varepsilon}^{\prime}+\lambda_{\varepsilon}^{\prime}\right) \lambda_{\varepsilon}^{\prime}+\left(\left(\omega_{\varepsilon}^{\prime} \lambda_{\varepsilon}-r_{\varepsilon}^{\prime}\right) \omega_{\varepsilon}^{\prime}\right) \lambda_{\varepsilon}\right] d s\right] d t \quad \forall \quad \tilde{t} \geq 1 / k \\
&=\int_{0}^{\infty} \vartheta \int_{0}^{1}\left[\left(r_{\varepsilon}^{\prime}-\lambda_{\varepsilon} \omega_{\varepsilon}^{\prime}\right)^{2}+\left(r_{\varepsilon} \omega_{\varepsilon}^{\prime}+\lambda_{\varepsilon}^{\prime}\right)^{2}\right] d s d t \quad \forall \quad \tilde{t} \geq 0,
\end{aligned}
$$

which is a weak in time formulation of (1.14).

The second equivalent formulation in (1.15) is then a consequence of the following computation

$$
\begin{aligned}
\left(z_{\varepsilon}^{\prime \prime \prime \prime}-\left(\frac{1}{\varepsilon} E^{\prime}\left(\left|z_{\varepsilon}^{\prime}\right|\right) \frac{z_{\varepsilon}^{\prime}}{\left|z_{\varepsilon}^{\prime}\right|}\right)^{\prime}\right)^{2} & =\left(\left(r_{\varepsilon} \frac{z_{\varepsilon}^{\prime \perp}}{\left|z_{\varepsilon}^{\prime}\right|}+\lambda_{\varepsilon} \frac{z_{\varepsilon}^{\prime}}{\left|z_{\varepsilon}^{\prime}\right|}\right)^{\prime}\right)^{2} \\
& =\left(r_{\varepsilon}^{\prime} \frac{z_{\varepsilon}^{\prime \perp}}{\left|z_{\varepsilon}^{\prime}\right|}+\lambda_{\varepsilon}^{\prime} \frac{z_{\varepsilon}^{\prime}}{\left|z_{\varepsilon}^{\prime}\right|}-r_{\varepsilon} \omega_{\varepsilon}^{\prime} \frac{z_{\varepsilon}^{\prime}}{\left|z_{\varepsilon}^{\prime}\right|}+\lambda_{\varepsilon} \omega_{\varepsilon}^{\prime} \frac{z_{\varepsilon}^{\prime \perp}}{\left|z_{\varepsilon}^{\prime}\right|}\right)^{2} \\
& =\left(r_{\varepsilon}^{\prime}+\lambda_{\varepsilon} \omega_{\varepsilon}^{\prime}\right)^{2}+\left(\lambda_{\varepsilon}^{\prime}-r_{\varepsilon} \omega_{\varepsilon}^{\prime}\right)^{2}
\end{aligned}
$$

which concludes the proof.

\section{Long time convergence}

One of the main tools in this section will be the fact that the best constant in the Poincaré-type inequality

$$
\int_{0}^{1} v^{2} d s \leq C \int_{0}^{1} v^{\prime 2} d s
$$

for $v \in H_{0}^{1}((0,1))$ is given by the reciprocal value of the first eigenvalue of the differential operator $v^{\prime \prime}$ in that space, $C=1 / \pi^{2}$.

As a first step in proving coercivity of $\mathcal{D}_{\varepsilon}$ with respect to $\mathcal{E}_{\varepsilon}$, we find the following lemma.

LEMMA 6.1. Let $\mathcal{E}_{\varepsilon}$ be the total energy defined in (1.1) and (1.10), respectively, and let $r_{\varepsilon}$ and $\lambda_{\varepsilon}$ be as defined in (1.12). Then there is a constant $C_{1}>0$ such that

$$
\mathcal{E}_{\varepsilon} \leq C_{1}\left(\left\|r_{\varepsilon}\right\|_{L_{s}^{2}}^{2}+\left\|\lambda_{\varepsilon}\right\|_{L_{s}^{2}}^{2}\right)
$$

Proof. We will treat the three different expressions in (1.10) separately. First observe that

$$
\int_{0}^{1}\left(b_{\varepsilon} \omega_{\varepsilon}^{\prime}\right)^{2} d \tilde{s} \leq \frac{1}{\pi^{2}} \int_{0}^{1}\left(\left(b_{\varepsilon} \omega_{\varepsilon}^{\prime}\right)^{\prime}\right)^{2} d \tilde{s} \leq \frac{b_{\max }^{2}}{\pi^{2}} \int_{0}^{1} r_{\varepsilon}^{2} d \tilde{s}
$$

which immediately implies that

$$
\left\|\omega_{\varepsilon}^{\prime}\right\|_{L^{2}}^{2} \leq \frac{b_{\max }^{2}}{\pi^{2} b_{\min }^{2}} \int_{0}^{1} r_{\varepsilon}^{2} d \tilde{s} .
$$

Next we use the definition of $\lambda_{\varepsilon}$ in (1.12), which we multiply by $\left(b_{\varepsilon}-1\right)$ obtaining $-\left(b_{\varepsilon}-1\right) b_{\varepsilon}^{\prime \prime} \leq\left(b_{\varepsilon}-1\right) \lambda_{\varepsilon}+b_{\varepsilon}\left(\omega_{\varepsilon}^{\prime}\right)^{2}$ since $(b-1) \frac{1}{\varepsilon} E^{\prime}\left(b_{\varepsilon}\right) \geq 0$, as $E$ is convex and takes its minimum at $x=1$. We integrate, and using $b_{\varepsilon}^{\prime}(0)=b_{\varepsilon}^{\prime}(1)=0$ we obtain 


$$
\begin{aligned}
\int_{0}^{1}\left(b_{\varepsilon}^{\prime}\right)^{2} d \tilde{s} & \leq \int_{0}^{1}\left[\left(b_{\varepsilon}-1\right) \lambda_{\varepsilon}+b_{\varepsilon}\left(\omega_{\varepsilon}^{\prime}\right)^{2}\right] d \tilde{s} \\
& \leq \max \left(b_{\max }-1,1-b_{\min }\right)\left\|\lambda_{\varepsilon}\right\|_{L^{2}}^{2}+b_{\max }\left\|\omega_{\varepsilon}^{\prime}\right\|_{L^{2}}^{2} .
\end{aligned}
$$

Finally, to obtain a control on $\int_{0}^{1} \frac{1}{\varepsilon} E\left(b_{\varepsilon}\right) d \tilde{s}$, we use Lemma 2.5 and (4.12), which imply that

$$
\frac{1}{\varepsilon}\left\|E^{\prime}\left(b_{\varepsilon}\right)\right\|_{L^{2}}^{2} \leq \varepsilon\left\|\lambda_{\varepsilon}-b_{\varepsilon}\left(\omega_{\varepsilon}^{\prime}\right)^{2}\right\|_{L^{2}}^{2}
$$

The fact that $\omega_{\varepsilon}^{\prime \prime}=\frac{r_{\varepsilon}-2 b_{\varepsilon}^{\prime} \omega_{\varepsilon}^{\prime}}{b_{\varepsilon}}$ allows us to estimate

$$
\begin{aligned}
\left\|\omega_{\varepsilon}^{\prime}\right\|_{L^{\infty}}^{2} & =\left\|\int_{0}^{s} \omega_{\varepsilon}^{\prime \prime} d \tilde{s}\right\|_{L^{\infty}}^{2} \leq\left\|\omega_{\varepsilon}^{\prime \prime}\right\|_{L^{1}}^{2} \\
& \leq \frac{2}{b_{\min }^{2}}\left(\left\|r_{\varepsilon}\right\|_{L^{1}}^{2}+4\left\|b_{\varepsilon}^{\prime}\right\|_{L^{2}}^{2}\left\|\omega_{\varepsilon}^{\prime}\right\|_{L^{2}}^{2}\right) \leq \frac{2}{b_{\min }^{2}}\left(\left\|r_{\varepsilon}\right\|_{L^{1}}^{2}+8 b_{\min }^{-2} \mathcal{E}_{I}\left\|b_{\varepsilon}^{\prime}\right\|_{L^{2}}^{2}\right),
\end{aligned}
$$

where we used (3.1). In summary, now using Lemma 2.5, we obtain

$$
\begin{aligned}
\int_{0}^{1} \frac{1}{\varepsilon} E\left(b_{\varepsilon}\right) d \tilde{s} & \leq \frac{1}{2 \kappa} \int_{0}^{1} \frac{1}{\varepsilon} E^{\prime}\left(b_{\varepsilon}\right)^{2} d \tilde{s} \leq \frac{1}{2 \kappa} \varepsilon\left\|\lambda_{\varepsilon}-b_{\varepsilon}\left(\omega_{\varepsilon}^{\prime}\right)^{2}\right\|_{L^{2}}^{2} \\
& \leq \varepsilon \frac{1}{\kappa}\left(\left\|\lambda_{\varepsilon}\right\|_{L^{2}}^{2}+b_{\max }^{2}\left\|\left(\omega_{\varepsilon}^{\prime}\right)^{2}\right\|_{L^{2}}^{2}\right) \leq \varepsilon \frac{1}{\kappa}\left(\left\|\lambda_{\varepsilon}\right\|_{L^{2}}^{2}+b_{\max }^{2}\left\|\omega_{\varepsilon}^{\prime}\right\|_{L^{\infty}}^{2}\left\|\omega_{\varepsilon}^{\prime}\right\|_{L^{2}}^{2}\right) \\
& \leq \varepsilon \frac{1}{\kappa}\left(\left\|\lambda_{\varepsilon}\right\|_{L^{2}}^{2}+2 b_{\min }^{-2} \mathcal{E}_{I} b_{\max }^{2}\left\|\omega_{\varepsilon}^{\prime}\right\|_{L^{\infty}}^{2}\right) .
\end{aligned}
$$

Now apply the inequalities (6.2), (6.3), and (6.5) combined with (6.4) to the total energy as formulated in (1.10) to obtain the result.

We finally conclude the exponential decay of the energy formulated in Theorem 1.4.

Proof. (Theorem 1.4.) We start with the result of Lemma 6.1 and obtain

$$
\begin{aligned}
\mathcal{E}_{\varepsilon} & \leq C_{1} \int_{0}^{1}\left(r_{\varepsilon}^{2}+\lambda_{\varepsilon}^{2}\right) d s \\
& =C_{1} \int_{0}^{1}\left[\left(z_{\varepsilon}^{\prime \prime \prime} \cdot \frac{z_{\varepsilon}^{\prime \perp}}{\left|z_{\varepsilon}^{\prime}\right|}\right)^{2}+\left(z_{\varepsilon}^{\prime \prime \prime} \cdot \frac{z_{\varepsilon}^{\prime}}{\left|z_{\varepsilon}^{\prime}\right|}-\frac{1}{\varepsilon} E\left(\left|z_{\varepsilon}^{\prime}\right|\right)\right)^{2}\right] d s \\
& =C_{1} \int_{0}^{1}\left|z_{\varepsilon}^{\prime \prime \prime}-\frac{1}{\varepsilon} E\left(\left|z_{\varepsilon}^{\prime}\right|\right) \frac{z_{\varepsilon}^{\prime}}{\left|z_{\varepsilon}^{\prime}\right|}\right|^{2} d s \\
& \leq \frac{C_{1}}{\pi^{2}} \int_{0}^{1}\left|\left(z_{\varepsilon}^{\prime \prime \prime}-\frac{1}{\varepsilon} E\left(\left|z_{\varepsilon}^{\prime}\right|\right) \frac{z_{\varepsilon}^{\prime}}{\left|z_{\varepsilon}^{\prime}\right|}\right)^{\prime}\right|^{2} d s=\frac{C_{1}}{\pi^{2}} \mathcal{D}_{\varepsilon},
\end{aligned}
$$

where we used (1.15). As a consequence, the energy dissipation $\mathcal{D}_{\varepsilon}$ is coercive with respect to the energy and and the following Poincaré-type inequality holds:

$$
\mathcal{D}_{\varepsilon} / \mathcal{E}_{\varepsilon} \geq \pi^{2} / C_{1},
$$


which implies directly the result.

As an immediate consequence of Theorem 1.4 we obtain the following refined bounds.

Corollary 6.2. Using the definitions in (1.16) we have

$$
\left\|\partial_{t} z_{\varepsilon}\right\|_{L_{t}^{2} L_{s}^{2}}^{2}=\left\|m_{\varepsilon}\right\|_{L_{t}^{2} L_{s}^{2}}^{2}+\left\|n_{\varepsilon}\right\|_{L_{t}^{2} L_{s}^{2}}^{2}=\mathcal{E}_{I} .
$$

As a consequence it holds that

$$
\left\|m_{\varepsilon}\right\|_{L_{t}^{2} L_{s}^{2}} \leq \sqrt{\mathcal{E}_{I}}, \quad\left\|n_{\varepsilon}\right\|_{L_{t}^{2} L_{s}^{2}} \leq \sqrt{\mathcal{E}_{I}} .
$$

We also obtain sharper bounds on the following quantities:

$$
\begin{aligned}
\left\|r_{\varepsilon}\right\|_{L_{t}^{2} L_{s}^{\infty}},\left\|\lambda_{\varepsilon}\right\|_{L_{t}^{2} L_{s}^{\infty}} & \leq \sqrt{\mathcal{E}_{I}}, \\
\left\|\omega_{\varepsilon}^{\prime} \lambda_{\varepsilon}\right\|_{L_{t}^{2} L_{s}^{2}},\left\|\omega_{\varepsilon}^{\prime} r_{\varepsilon}\right\|_{L_{t}^{2} L_{s}^{2}} & \leq \sqrt{2} \mathcal{E}_{I} b_{\min }^{-1}, \quad \text { and } \\
\left\|\lambda_{\varepsilon}^{\prime}\right\|_{L_{t}^{2} L_{s}^{2}},\left\|r_{\varepsilon}^{\prime}\right\|_{L_{t}^{2} L_{s}^{2}} & \leq \sqrt{2} \mathcal{E}_{I} b_{\min }^{-1}+\sqrt{\mathcal{E}_{I}} .
\end{aligned}
$$

Proof. Integrate (1.14) with respect to time and combine it with (4.10). Using this result we go again through the estimates in the proof of Lemma 4.2.

\section{Convergence as $\varepsilon \rightarrow 0$}

Lemma 7.1. Let $\left(\omega_{\varepsilon}, b_{\varepsilon}, \lambda_{\varepsilon}\right)$ be a solution to (1.13) according to Theorem 1.2. Then there is $\omega_{0}$ such that $\omega_{\varepsilon} \rightarrow \omega_{0}$ in $L^{1}\left((0, T), W^{2, r}(0,1)\right) \cap L^{q}\left((0, T), H^{1}(0,1)\right), 1 \leq r, q<$ $\infty$.

Proof. We obtain the strong convergence of $\omega_{\varepsilon}$ by an application of the LionsAubin Lemma as formulated in [12]. On the one hand, using (1.17), observe that the family of distributions $\left\{\partial_{t} \omega_{\varepsilon}\right\}$ is uniformly bounded in $L^{1}\left(0, T ; H^{-1}((0,1))\right)$ as

$$
\begin{aligned}
\left\langle\partial_{t} \omega_{\varepsilon}, v\right\rangle_{H_{s}^{-1}} & =\int_{0}^{1}\left[n_{\varepsilon}\left(\frac{v^{\prime}}{b_{\varepsilon}}-\frac{v}{b_{\varepsilon}^{2}} b_{\varepsilon}^{\prime}\right)+m_{\varepsilon} \omega_{\varepsilon}^{\prime} \frac{v}{b_{\varepsilon}}\right] d s \\
& \leq \frac{1}{b_{\min }}\left(\int _ { 0 } ^ { 1 } \left|n_{\varepsilon}\left\|v^{\prime}\left|d s+\frac{\|v\|_{L_{s}^{\infty}}}{b_{\min }} \int_{0}^{1}\right| n_{\varepsilon}|| b_{\varepsilon}^{\prime}\left|d s+\|v\|_{L_{s}^{\infty}} \int_{0}^{1}\right| \omega_{\varepsilon}^{\prime}|| m_{\varepsilon} \mid d s\right)\right.\right.
\end{aligned}
$$

for all $v \in H_{0}^{1}((0,1))$. This implies

$$
\begin{gathered}
\int_{0}^{T}\left|\sup _{v \in H_{0}^{1}} \frac{\left\langle\partial_{t} \omega_{\varepsilon}, v\right\rangle_{H_{s}^{-1}}}{\|v\|_{H_{0}^{1}}}\right| d t \\
\leq \sup _{v \in H_{0}^{1}} \frac{1}{b_{\min }\|v\|_{H_{0}^{1}}}\left(\int_{0}^{T} \int_{0}^{1}\left|n_{\varepsilon} \| v^{\prime}\right| d s d t+\frac{\|v\|_{L_{s}^{\infty}}}{b_{\min }} \int_{0}^{T} \int_{0}^{1}\left|n_{\varepsilon}\right|\left|b_{\varepsilon}^{\prime}\right| d s d t\right. \\
\left.+\|v\|_{L_{s}^{\infty}} \int_{0}^{T} \int_{0}^{1}\left|\omega_{\varepsilon}^{\prime} \| m_{\varepsilon}\right| d s d t\right) \\
\leq \frac{1}{b_{\min }}\left(\sqrt{T}\left\|n_{\varepsilon}\right\|_{L^{2}\left(0, T ; L_{s}^{2}\right)}+\frac{1}{b_{\min }}\left\|b_{\varepsilon}^{\prime}\right\|_{L^{2}\left(0, T ; L_{s}^{2}\right)}\left\|n_{\varepsilon}\right\|_{L^{2}\left(0, T ; L_{s}^{2}\right)}\right. \\
\left.+\left\|\omega_{\varepsilon}^{\prime}\right\|_{L^{2}\left(0, T ; L_{s}^{2}\right)}\left\|m_{\varepsilon}\right\|_{L^{2}\left(0, T ; L_{s}^{2}\right)}\right)
\end{gathered}
$$




$$
\leq \frac{\sqrt{T}}{b_{\min }}\left(\left\|n_{\varepsilon}\right\|_{L^{2}\left(0, T ; L_{s}^{2}\right)}+\sqrt{2 \mathcal{E}_{I}}\left(\frac{1}{b_{\min }}\left\|n_{\varepsilon}\right\|_{L^{2}\left(0, T ; L_{s}^{2}\right)}+b_{\min }^{-2}\left\|m_{\varepsilon}\right\|_{L^{2}\left(0, T ; L_{s}^{2}\right)}\right)\right) .
$$

Here we used the uniform bounds of (3.1) and Corollary 6.2.

Since the family $\left\{\omega_{\varepsilon}\right\}$ is uniformly bounded in $L^{1}\left(0, T ; W^{3,1}(0,1)\right)$ by Lemma 4.3 , and due to the compact embedding $W^{1,1}((0,1)) \hookrightarrow L^{r}((0,1))(1 \leq r<\infty)$, it holds that the family $\omega_{\varepsilon}$ is relatively compact in $L^{1}\left(0, T ; W^{2, r}(0,1)\right)$.

By interpolation, using the uniform bound in $\left.L_{t}^{\infty} H^{1}(0,1)\right)$ on $\omega_{\varepsilon}$, this also holds true in $L^{q}\left(0, T ; H^{1}(0,1)\right)$ for any $1 \leq q<\infty$.

Lemma 7.2. Let $\left(\omega_{\varepsilon}, b_{\varepsilon}, \lambda_{\varepsilon}\right)$ be a solution to (1.13) according to Theorem 1.2. Then it holds that $b_{\varepsilon} \rightarrow \mathbf{1}$ in $L^{1}\left((0, T), W^{1, r}(0,1)\right) \cap L^{q}\left((0, T), H^{1}(0,1)\right), 1 \leq r, q<\infty$.

Proof. We obtain the strong convergence of $b_{\varepsilon}$ by an application of the LionsAubin Lemma as formulated in [12]. On the one hand, using (1.13) and the definitions (1.16), observe that the family of distributions $\left\{\partial_{t} b_{\varepsilon}\right\}$ is uniformly bounded in $L^{1}\left(0, T ; H^{-1}((0,1))\right)$ as

$$
\left\langle\partial_{t} b_{\varepsilon}, v\right\rangle_{H_{s}^{-1}}=\int_{0}^{1}\left[-m_{\varepsilon} v^{\prime}+n_{\varepsilon} \omega_{\varepsilon}^{\prime} v\right] d s \leq \int_{0}^{1}\left|m_{\varepsilon}\right|\left|v^{\prime}\right| d s+\|v\|_{L_{s}^{\infty}} \int_{0}^{1}\left|\omega_{\varepsilon}^{\prime} \| n_{\varepsilon}\right| d s
$$

for all $v \in H_{0}^{1}((0,1))$. This implies

$$
\begin{aligned}
& \int_{0}^{T}\left|\sup _{v \in H_{0}^{1}} \frac{\left\langle\partial_{t} b_{\varepsilon}, v\right\rangle_{H_{s}^{-1}}}{\|v\|_{H_{0}^{1}}}\right| d t \\
\leq & \sup _{v \in H_{0}^{1}} \frac{1}{\|v\|_{H_{0}^{1}}}\left(\int_{0}^{T} \int_{0}^{1}\left|m_{\varepsilon}\left\|v^{\prime}\left|d s d t+\|v\|_{L_{s}^{\infty}} \int_{0}^{T} \int_{0}^{1}\right| \omega_{\varepsilon}^{\prime}\right\| n_{\varepsilon}\right| d s d t\right) \\
\leq & \int_{0}^{T}\left\|m_{\varepsilon}\right\|_{L_{s}^{2}} d t+\left\|\omega_{\varepsilon}^{\prime}\right\|_{L^{2}\left(0, T ; L_{s}^{2}\right)}\left\|n_{\varepsilon}\right\|_{L^{2}\left(0, T ; L_{s}^{2}\right)} \\
\leq & \sqrt{T}\left\|n_{\varepsilon}\right\|_{L^{2}\left(0, T ; L_{s}^{2}\right)}+\sqrt{T 2 b_{\min }^{-2} \mathcal{E}_{I}}\left\|m_{\varepsilon}\right\|_{L^{2}\left(0, T ; L_{s}^{2}\right)} .
\end{aligned}
$$

Here we used the uniform bounds of (3.1) and Corollary 6.2.

Since the family $\left\{b_{\varepsilon}\right\}$ is uniformly bounded in $L^{1}\left(0, T ; W^{2,1}(0,1)\right)$ by Lemma 4.3 , and due to the compact embedding $W^{1,1}((0,1)) \hookrightarrow L^{r}((0,1))(1 \leq r<\infty)$, it holds that the family $b_{\varepsilon}$ is relatively compact in $L^{1}\left(0, T ; W^{1, r}(0,1)\right)$.

By interpolation, using the uniform bound in $\left.L_{t}^{\infty} H^{1}(0,1)\right)$ on $b_{\varepsilon}$, this holds also true in $L^{q}\left(0, T ; H^{1}(0,1)\right)$ for any $1 \leq q<\infty$.

Finally, we consider the first inequality formulated in Lemma 2.5 and obtain as a consequence of energy dissipation (1.14) that

$$
\left\|b_{\varepsilon}(t, .)-\mathbf{1}\right\|_{L_{s}^{2}} \leq \frac{2}{\kappa} \sqrt{\varepsilon \mathcal{E}_{I}} \text { for all } t>0,
$$

which allows us to identify the limit of any strongly converging subsequence of the family $\left\{b_{\varepsilon}\right\}$ as the constant function $\mathbf{1}$.

Lemma 7.3. Let $\left(\omega_{\varepsilon}, b_{\varepsilon}, \lambda_{\varepsilon}\right)$ be a solution to (1.13) according to Theorem 1.2. Then there are subsequences and limit functions such that $\lambda_{\varepsilon} \rightarrow \lambda_{0}$ in $L_{t}^{2} H_{s}^{1}$ and $r_{\varepsilon} \rightarrow \omega_{0}^{\prime \prime}$ in $L^{2}\left((0, T), L_{s}^{2}\right)$, with $r_{\varepsilon} \rightarrow \omega_{0}^{\prime \prime}$ in $L_{t}^{2} H_{s}^{1}$. 
Proof. The strong convergence of $\omega_{\varepsilon}$ and $b_{\varepsilon}$ (Lemma 7.1 and 7.2) imply the strong convergence of $r_{\varepsilon}$ to $\omega_{0}^{\prime \prime}$. The weak convergence of $r_{\varepsilon}^{\prime}$ and also the weak convergence of $\lambda_{\varepsilon}$ to a limit function $\lambda_{0}$ are a consequence of the uniform bounds stated in Corollary 6.2.

Finally we finish with the proof of Theorem 1.5, which summarizes the results of lemmas 7.1, 7.2, and 7.3 and states the consistency of the model (1.5) with the limit model (1.6) and, as a consequence, the strong convergence of $m_{\varepsilon}$ and $n_{\varepsilon}$.

Proof. (Theorem 1.5) The convergence results of lemmas 7.1, 7.2, and 7.3 immediately allow us to pass to the limit as $\varepsilon \rightarrow 0$ in the system (1.20), (1.21), obtaining (2.2), (2.3) as the limit system.

Observe that the definitions (1.16), together with the strong convergence of $\omega_{\varepsilon}$ (Lemma 7.1) and the weak convergence of $r_{\varepsilon}$ and $\lambda_{\varepsilon}$ (Lemma 7.3), imply that

$$
m_{\varepsilon} \rightarrow \omega_{0}^{\prime} \omega_{0}^{\prime \prime}+\lambda_{0}^{\prime} \quad \text { and } \quad n_{\varepsilon} \rightarrow \omega_{0}^{\prime \prime \prime}-\omega_{0}^{\prime} \lambda_{0} \quad \text { in } L_{t}^{2} L_{s}^{2} .
$$

The fact that the equality (6.8) also holds in the limit as $\varepsilon_{j} \rightarrow 0,(2.7)$, implies

$$
\left\|m_{\varepsilon}\right\|_{L_{t}^{2} L_{s}^{2}}^{2}+\left\|n_{\varepsilon}\right\|_{L_{t}^{2} L_{s}^{2}}^{2}=\mathcal{E}_{I}=\left\|\omega_{0}^{\prime} \omega_{0}^{\prime \prime}+\lambda_{0}^{\prime}\right\|_{L_{t}^{2} L_{s}^{2}}^{2}+\left\|\omega_{0}^{\prime \prime \prime}-\omega_{0}^{\prime} \lambda_{0}\right\|_{L_{t}^{2} L_{s}^{2}}^{2}
$$

These two facts imply the strong convergence of $m_{\varepsilon}$ and $n_{\varepsilon}$ since

$$
\begin{aligned}
& \int_{0}^{\infty} \int_{0}^{1}\left[\left(m_{\varepsilon}-m_{0}\right)^{2}+\left(n_{\varepsilon}-n_{0}\right)^{2}\right] d s d t \\
= & \int_{0}^{\infty} \int_{0}^{1}\left[m_{\varepsilon}^{2}-2 m_{\varepsilon} m_{0}+m_{0}^{2}+n_{\varepsilon}^{2}-2 n_{\varepsilon} n_{0}+n_{0}^{2}\right] d s d t \rightarrow 0 \quad \text { as } \quad \varepsilon \rightarrow 0 .
\end{aligned}
$$

Acknowledgment. This work has been supported by the Vienna Science and Technology Fund (WWTF) through the project "Mathematical modeling of actin driven cell migration" of C. Schmeiser.

\section{REFERENCES}

[1] L. Ambrosio, N. Gigli, and G. Savaré, Gradient Flows in Metric Spaces and in the Space of Probability Measures, Lectures in Mathematics ETH Zürich, Birkhäuser Verlag, Basel, 2005.

[2] C. Bost, G.H. Cottet, and E. Maitre, Convergence analysis of a penalization method for the three-dimensional motion of a rigid body in an incompressible viscous fluid, SIAM J. Num. Anal., 48(4), 1313-1337, 2010.

[3] E. Casas, M. Mateos, and J.-P. Raymond, Penalization of Dirichlet optimal control problems, ESAIM Control Optim. Calc. Var., 15(4), 782-809, 2009.

[4] E. De Giorgi, A. Marino, and M. Tosques, Problems of evolution in metric spaces and maximal decreasing curve, Atti Accad. Naz. Lincei Rend. Cl. Sci. Fis. Mat. Natur., 68(8), 180-187, 1980.

[5] E. Harold Dill, Kirchhoff's theory of rods, Arch. Hist. Exact Sci., 44(1), 1-23 (English), 1992.

[6] X.X. Huang and X.Q. Yang, Convergence analysis of a class of nonlinear penalization methods for constrained optimization via first-order necessary optimality conditions, J. Optim. Theo. Appl., 116, 311-332, 2003.

[7] G.R. Kirchhoff, Über das Gleichgewicht und die Bewegung eines unendlich dünnen elastischen Stabes, J. Reine. Angew. Math., 56, 285-313, 1859.

[8] A. Linnér, Some properties of the curve straightening flow in the plane, Trans. Amer. Math. Soc., 314(2), 605-618, 1989.

[9] D. Oelz, On the curve straightening flow of inextensible, open, planar curves, S $\vec{e} \mathrm{MA}$ J., 54, $5-24,2010$. 
[10] D. Oelz and C. Schmeiser, Cell Mechanics: From Single Scale-based Models to Multiscale Modelling, Mathematical Modelling of Cytoskeleton Dynamics and Cell Migration, Chapman and Hall/CRC Press, 2010.

[11] D. Oelz and C. Schmeiser, Simulation of lamellipodial fragments, J. Math. Biol., 64(3), 513-528, 2012.

[12] J. Simon, Compact sets in the space $L^{p}(0, T ; B)$, Ann. Mat. Pura. Appl., 146(4), 65-96, 1987. 